\title{
Behavior in Strategic Settings: Evidence from a Million Rock-Paper-Scissors Games
}

\author{
Dimitris Batzilis ${ }^{1,+} \mathbb{C}$, Sonia Jaffe ${ }^{2, *,+} \mathbb{C}$, Steven Levitt ${ }^{3,+}$, John A. List ${ }^{3,+}$ and Jeffrey Picel ${ }^{4,+}$ \\ 1 Department of Economics, American College of Greece-Deree, Agia Paraskevi 15342, Greece; \\ dbatzilis@acg.edu \\ 2 Office of the Chief Economist, Microsoft, Redmond, WA 98052, USA \\ 3 Department of Economics, University of Chicago, Chicago, IL 60637, USA; slevitt@uchicago.edu (S.L.); \\ jlist@uchicago.edu (J.A.L.) \\ 4 Department of Economics, Harvard University, Cambridge, MA 02138, USA; jpicel@fas.harvard.edu \\ * Correspondence: spj@uchicago.edu \\ + These authors contributed equally to this work.
}

Received: 6 February 2019; Accepted: 4 April 2019; Published: 10 April 2019

\begin{abstract}
We make use of data from a Facebook application where hundreds of thousands of people played a simultaneous move, zero-sum game-rock-paper-scissors-with varying information to analyze whether play in strategic settings is consistent with extant theories. We report three main insights. First, we observe that most people employ strategies consistent with Nash, at least some of the time. Second, however, players strategically use information on previous play of their opponents, a non-Nash equilibrium behavior; they are more likely to do so when the expected payoffs for such actions increase. Third, experience matters: players with more experience use information on their opponents more effectively than less experienced players, and are more likely to win as a result. We also explore the degree to which the deviations from Nash predictions are consistent with various non-equilibrium models. We analyze both a level- $k$ framework and an adapted quantal response model. The naive version of each these strategies - where players maximize the probability of winning without considering the probability of losing-does better than the standard formulation. While one set of people use strategies that resemble quantal response, there is another group of people who employ strategies that are close to $k_{1}$; for naive strategies the latter group is much larger.
\end{abstract}

Keywords: play in strategic settings; large-scale data set; Nash equilibrium; non-equilibrium strategies

JEL Classification: C72; D03

Over the last several decades game theory has profoundly altered the social science landscape. Across economics and its sister sciences, elements of Nash equilibrium are included in nearly every analysis of behavior in strategic settings. For their part, economists have developed deep theoretical insights into how people should behave in a variety of important strategic environments-from optimal actions during wartime to more mundane tasks such as how to choose a parking spot at the mall. These theoretical predictions of game theory have been tested in lab experiments (e.g., see cites in $[1,2])$, and to a lesser extent in the field (e.g., [3-6] and cites therein).

In this paper, we take a fresh approach to studying strategic behavior outside the lab, exploiting a unique dataset that allows us to observe play while the information shown to the player changes. 
In particular, we use data from over one million matches of rock-paper-scissors (RPS) ${ }^{1}$ played on a historically popular Facebook application. Before each match (made up of multiple throws), players are shown a wealth of data about their opponent's past history: the percent of past first throws in a match that were rock, paper, or scissors, the percent of all throws that were rock, paper, or scissors, and all the throws from the opponents' most recent five games. These data thus allow us to investigate whether, and to what extent, players' strategies incorporate this information.

The informational variation makes the strategy space for the game potentially much larger than a one-shot RPS game. However, we show that in Nash equilibrium, players must expect their opponents to mix equally across rock-paper-scissors-same as in the one-shot game. Therefore, a player has no use for information on her opponent's history when her opponent is playing Nash.

To the extent that an opponent systematically deviates from Nash; however, knowledge of that opponent's history can potentially be exploited.2,3 Yet it is not obvious how one should use the information provided. Players can use the information to determine whether an opponent's past play is consistent with Nash, but without seeing what information an opponent was reacting to (they do not observe the past histories of the opponent's previous opponents), it is hard to guess what non-Nash strategy the opponent may be using. Additionally, players are not shown information about their own past play, so if a player wants to exploit an opponent's expected reaction, he must keep track of his own history of play.

Because of the myriad of possible responses, we start with a reduced-form analysis of the first throw in each match to describe how players respond to the provided information. We find that players use information: for example, they are more likely to play rock when their opponent has played more scissors (which rock beats) or less paper (which beats rock) on previous first throws, though the latter effect is smaller. When we do the analysis at the player level, $47 \%$ of players are reacting to information about their opponents' history in a way that is statistically significant. ${ }^{4}$ Players also have a weak negative correlation across their own first throws.

This finding motivated us to adopt a structural approach to evaluate the performance of two well-known alternatives to Nash equilibrium: level- $k$ and quantal response. The level- $k$ model posits that players are of different types according to the depth of their reasoning about the strategic behavior of their opponents [15-18]. Players who are $k_{0}$ do not respond to information available about their opponent. This can either mean that they play randomly (e.g., [19]) or that they play some focal or salient strategy (e.g., [20,21]). Players who are $k_{1}$ respond optimally to a $k_{0}$ player, which in our context means responding to the focal strategy of the opponent's (possibly skewed) historical distribution of throws; $k_{2}$ players respond optimally to $k_{1}$, etc. ${ }^{5}$

Level- $k$ theory acknowledges the difficulty of calculating equilibria and of forming equilibrium beliefs, especially in one-shot games. It has been applied to a variety of laboratory games (e.g., [19,20,22-24]) and some naturally occurring environments (e.g., [25-29]). This paper has substantially more data than most other level- $k$ studies, both in number of observations and in the richness of the information structure. As suggested by an anonymous referee and acknowledged

1 Two players each play rock, paper, or scissors. Rock beats scissors; scissors beats paper; paper beats rock. If they both play the same, it is a tie. The payoff matrix is in Section 2.

2 If the opponent is not playing Nash, then Nash is no longer a best response. In symmetric zero-sum games such as RPS, deviating from Nash is costless if the opponent is playing Nash (since all strategies have an expected payoff of zero), but if a player thinks he knows what non-Nash strategy his opponent is using then there is a profitable deviation from Nash.

3 Work in evolutionary game theory on RPS has looked at how the population's distribution of strategies evolves towards or around Nash equilibrium (e.g., $[7,8])$. Past work on fictitious play has showed that responding to the opponents' historical frequency of strategies leads to convergence to Nash equilibrium [9-11]. Young [12] also studies how conventions evolve as players respond to information about how their opponents have behaved in the past, while Mookherjee and Sopher $[13,14]$ examine the effect of information on opponents' history on strategic choices.

4 When doing a test at the player level, we expect about $5 \%$ of players to be false positives, so we take these numbers as evidence on behavior only when they are statistically significant for substantially more than $5 \%$ of players.

5 Since the focal $k_{0}$ strategies can be skewed, our $k_{1}$ and $k_{2}$ strategies usually designate a unique throw, which would not be true if $k_{0}$ were constrained to be a uniform distribution. 
in Ho et al. [24], the implication of the fictitious play learning rule is that players should employ a $k_{1}$ strategy, best responding to the historical frequency of their opponents' plays on the assumption that it predicts their future choices. When $k_{1}$ play is defined as the best response to past historical play, as in the current context, it is of course indistinguishable from fictitious play.

We adapt level- $k$ theory to our repeated game context. Empirically, we use maximum likelihood to estimate how often each player plays $k_{0}, k_{1}$, and $k_{2}$, assuming that they are restricted to those three strategies. We find that most of the play is best described as $k_{0}$ (about $74 \%$ ). On average, $k_{1}$ is used in $18.5 \%$ of throws. The average $k_{2}$ estimate is $7.7 \%$, but for only $12 \%$ of players do we reject at the $95 \%$ level that they never play $k_{2}$. Most players use a mixture of strategies, mainly $k_{0}$ and $k_{1}{ }^{6}$ We also find that $20 \%$ of players deviate significantly from $\frac{1}{3}, \frac{1}{3}, \frac{1}{3}$ when playing $k_{0}$. We also consider a cognitive hierarchy version of the model and a naive version where players maximize the probability of winning without worrying about losing. The rates of play of the analogous strategies are similar to the baseline level- $k$, but the naive level- $k$ is a better fit for most players.

We also show that play is more likely to be consistent with $k_{1}$ when the expected return to $k_{1}$ is higher. This effect is larger when the opponent has a longer history-that is, when the skewness in history is less likely to be noise. The fact that players respond to the level of the perceived expected $\left(k_{1}\right)$ payoff, not just whether it is the highest payoff, is related to the idea of quantal response: that players' probability of using a pure strategy is increasing in the relative perceived expected payoff of that strategy. ${ }^{7}$ This can be thought of as a more continuous version of a $k_{1}$ strategy. Rather than always playing the strategy with the highest expected payoff as under $k_{1}$, the probability of playing a strategy increases with the expected payoff. As the random error in this (non-equilibrium) quantal response approaches zero (or the responsiveness of play to the expected payoff goes to infinity) this converges to the $k_{1}$ strategy. On average, we find that increasing the expected payoff to a throw by one standard deviation increases the probability it is played by 7.3 percentage points (more than one standard deviation). The coefficient is positive and statistically significant for $63 \%$ of players.

If players were using the $k_{1}$ strategy, we would also find that expected payoffs have a positive effect on probability of play. Similarly, if players used quantal response, many of their throws would be consistent with $k_{1}$ and our maximum likelihood analysis would indicate some $k_{1}$ play. The above evidence does not allow us to state which model is a better fit for the data. To test whether $k_{1}$ or quantal response better explains play, we compare the model likelihoods. The quantal response model is significantly better than the $k_{1}$ model for 18.3 percent of players, yet the $k_{1}$ model is significantly better for 17.5 percent of players. We interpret this result as suggesting that there are some players whose strategies are close to $k_{1}$, or fictitious play, and a distinct set of players whose strategies resemble quantal response. We also compare naive level- $k$ to a naive version of the quantal response model. Here level- $k$ does better. About $12 \%$ of players significantly favor the quantal response model and $26 \%$ significantly favor the naive level- $k$. The heterogeneity in player behavior points to the value of studies such as this one that have sufficient data to do within player analyses. In sum, our data paint the picture that there is a fair amount of equilibrium play, and when we observe non-Nash play, extant models have some power to explain the data patterns.

The remainder of the paper is structured as follows. Section 1 describes the Facebook application in which the game is played and presents summary statistics of the data. Section 2 describes the theoretical model underlying the game, and the concept and implications of Nash equilibrium in this setting. Section 3 explores how players respond to the information about their opponents' histories.

6 As we discuss in Section 4, there are several reasons that may explain why we find lower estimates for $k_{1}$ and $k_{2}$ play than in previous work. Many players may not remember their own history, which is necessary for playing $k_{2}$. Also, given that $k_{0}$ is what players would most likely play if they were not shown the information (i.e., when they play RPS outside the application), it may be more salient than in other contexts.

7 Because we think players differ in the extent to which they respond to information and consider expected payoffs, we do not impose the restriction from quantal response equilibrium theory [30] that the perceived expected payoffs are correct. Instead, we require that the expected payoffs are calculated based on the history of play. See Section 5 for more detail. 
Section 4 explains how we adapt level- $k$ theory to this context and provides parameter estimates. Section 5 adapts a non-equilibrium version of the quantal response model to our setting. Section 6 compares the level- $k$ and quantal response models. Section 7 concludes.

\section{Data: Roshambull}

RPS, also known as Rochambeau and jan-ken-pon, is said to have originated in the Chinese Han dynasty, making its way to Europe in the 18th century. To this day, it continues to be played actively around the world. There is even a world RPS championship sponsored by Yahoo. ${ }^{8}$

The source of our data is an early Facebook 'app' called Roshambull. (The name is a combination of Rochambeau and the name of the firm sponsoring the app, Red Bull.) Roshambull allowed users to play RPS against other Facebook users - either by challenging a specific person to a game or by having the software pair them with an opponent. It was a very popular app for its era with 340,213 users $(\approx 1.7 \%$ of Facebook users in 2007$)$ starting at least one match in the first three months of the game's existence. Users played best-two-out-of-three matches for prestige points known as 'creds'. They could share their records on their Facebook page and there was a leader board with the top players' records.

To make things more interesting for players, before each match the app showed them a "scouting sheet" with information on the opponent's history of play. ${ }^{9}$ In particular, the app showed each player the opponent's distribution of throws on previous first throws of a match (and the number of matches) and on all previous throws (and the number of throws), as well as a play-by-play breakdown of the opponent's previous five matches. It also shows the opponent's win-loss records and the number of creds wagered. Figure 1 shows a sample screenshot from the game.

Our dataset contains 2,636,417 matches, all the matches played between 23 May 2007 (when the program first became available to users) and 14 August 2007. For each throw, the dataset contains a player ID, match number, throw number, throw type, and the time and date at which the throw was made. ${ }^{10}$ This allows us to create complete player histories at each point in time. Most players play relatively few matches in our three month window: the median number of matches is 5 and the mean is $15 .{ }^{11}$ Figure 2 shows the distribution of the number of matches a player played.

Some of our inference depends upon having many observations per player; for those sections, our analysis is limited to the 7758 "experienced" players for whom we observe at least 100 clean matches. They play an average of 192 matches; the median is 148 and the standard deviation is $139 .{ }^{12}$ Because these are the most experienced players, their strategies may not be representative; one might expect more sophisticated strategies in this group relative to the Roshambull population as a whole.

Table 1 summarizes the play and opponents' histories shown in the first throw of each match, for both the entire sample and the experienced players. For all the empirical analysis we focus on the first throw in each match. Modeling non-equilibrium behavior on subsequent throws is more

8 RPS is usually played for low stakes, but sometimes the result carries with it more serious ramifications. During the World Series of Poker, an annual $\$ 500$ per person RPS tournament is held, with the winner taking home $\$ 25,000$. RPS was also once used to determine which auction house would have the right to sell a $\$ 12$ million Cezanne painting. Christie's went to the 11-year-old twin daughters of an employee, who suggested "scissors" because "Everybody expects you to choose 'rock'." Sotheby's said that they treated it as a game of chance and had no particular strategy for the game, but went with "paper" [31].

9 Bart Johnston, one of the developers said, "We've added this intriguing statistical aspect to the game... You're constantly trying to out-strategize your opponent" [32].

10 Unfortunately we only have a player id for each player; there is no demographic information or information about their out-of-game connections to other players.

11 Because of the possibility for players to collude to give one player a good record if the other does not mind having a bad one, we exclude matches from the small fraction of player-pairs for which one player won an implausibly high share of the matches ( $100 \%$ of $\geq 10$ games or $80 \%$ of $\geq 20$ games). To accurately recreate the information that opponents were shown when those players played against others, we still include those "collusion" matches when forming the players' histories.

12 Depending on the opponent's history, the strategies we look at may not indicate a unique throw (e.g., if rock and paper have the same expected payoffs); for some analyses we only use players who have 100 clean matches where the strategies being considered indicate a unique throw, so we use between 5405 and 7758 players. 
complicated because in addition to their opponent's history, a player may also respond to the prior throws in the match.

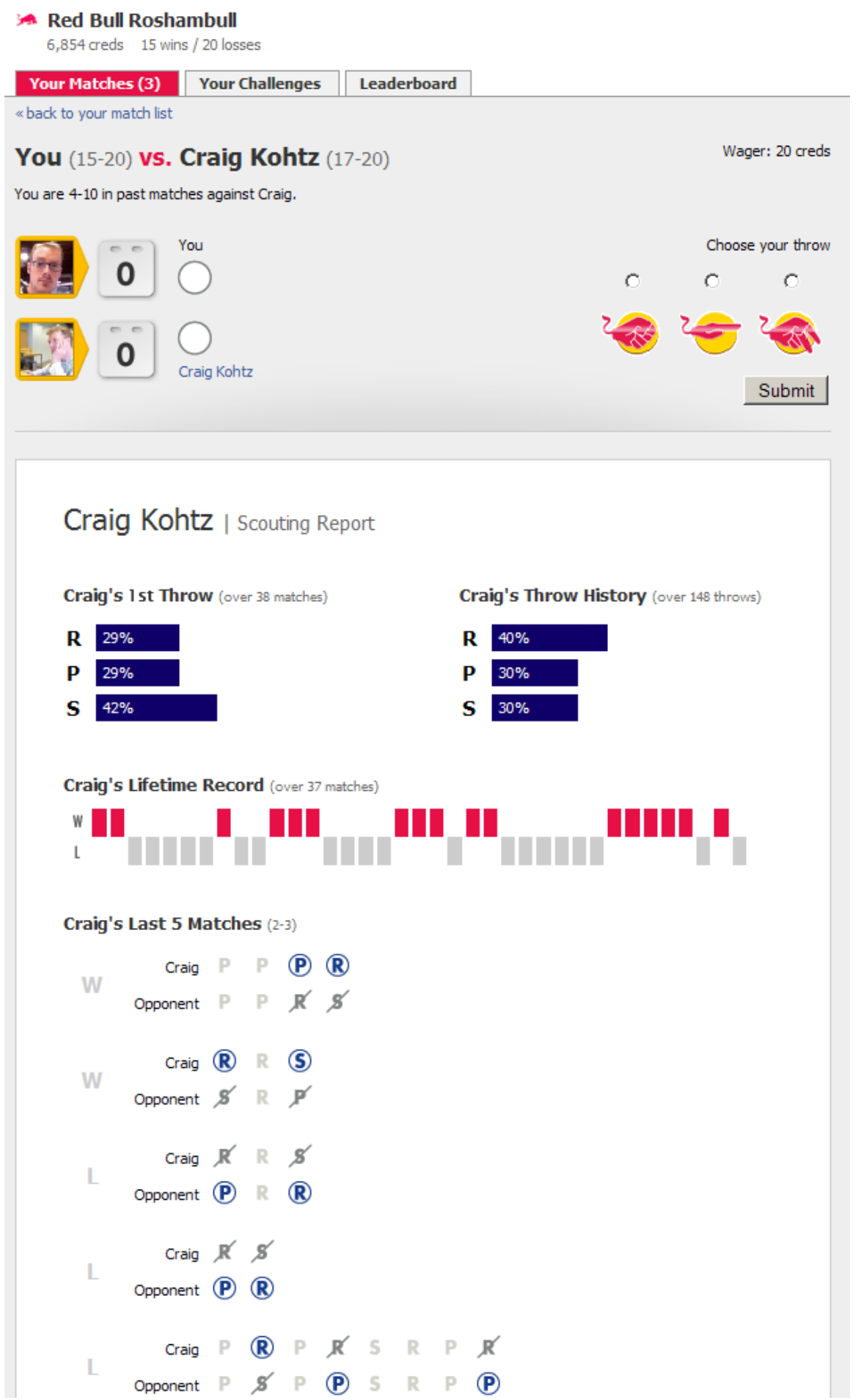

Craig Kohtz | Scouting Report

$\begin{array}{ll}\text { Craig's 1 st Throw (over } 38 \text { matches) } & \text { Craig's Throw History (over 148 throws) } \\ \text { R } 29 \% & \text { R } 40 \% \\ \text { P } 29 \% & \text { P } 30 \% \\ \text { S } 42 \% & \text { S } 30 \%\end{array}$

Craig's Lifetime Record (over 37 matches)

w

ᄂ

Craig's Last 5 Matches (2-3)

Figure 1. Screenshot of the Roshambull App. 


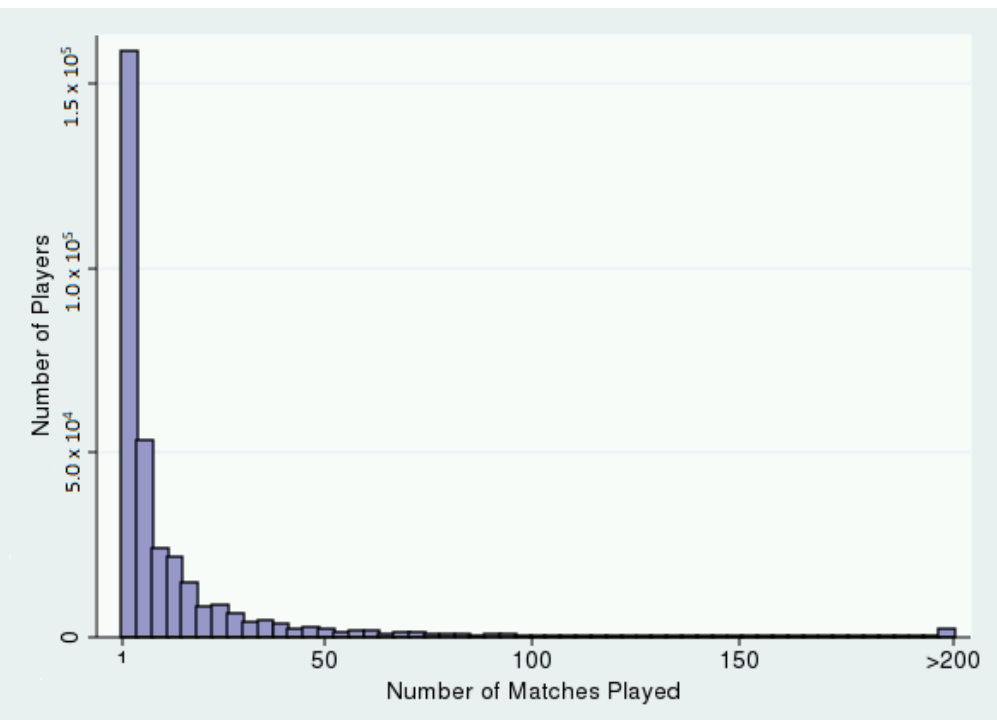

Figure 2. Number of matches played by Roshambull users. Note: The figure shows the number of clean matches played by the 334,661 players who had at least one clean, completed match (see Footnote 11 for a description of the data cleaning). The data has a very long right tail, so all the players with over 200 matches are grouped together in the right-most bar.

Table 1. Summary Statistics of First Throws.

\begin{tabular}{lccccc}
\hline \multirow{2}{*}{ Variable } & \multicolumn{2}{c}{ Full Sample } & & \multicolumn{2}{c}{ Restricted Sample } \\
\cline { 2 - 3 } & Mean & (SD) & & Mean & (SD) \\
\hline Throw Rock (\%) & 33.99 & $(47.37)$ & & 32.60 & $(46.87)$ \\
Throw Paper (\%) & 34.82 & $(47.64)$ & & 34.56 & $(47.56)$ \\
Throw Scissors (\%) & 31.20 & $(46.33)$ & & 32.84 & $(46.96)$ \\
Player's Historical \%Rock & 34.27 & $(19.13)$ & & 32.89 & $(8.89)$ \\
Player's Historical \%Paper & 35.14 & $(18.80)$ & & 34.88 & $(8.97)$ \\
Player's Historical \%Scissors & 30.59 & $(17.64)$ & & 32.23 & $(8.57)$ \\
Opp's Historical \%Rock & 34.27 & $(19.13)$ & & 33.59 & $(13.71)$ \\
Opp's Historical \%Paper & 35.14 & $(18.80)$ & & 34.76 & $(13.46)$ \\
Opp's Historical \%Scissors & 30.59 & $(17.64)$ & 31.65 & $(12.84)$ \\
Opp's Historical Skew & 10.42 & $(18.08)$ & & 5.39 & $(12.43)$ \\
Opp's Historical \%Rock (all throws) & 35.45 & $(12.01)$ & & 34.81 & $(8.59)$ \\
Opp's Historical \%Paper (all throws) & 34.01 & $(11.74)$ & & 34.10 & $(8.35)$ \\
Opp's Historical \%Scissors (all throws) & 30.54 & $(11.07)$ & 31.09 & $(7.98)$ \\
Opp's Historical Length (matches) & 55.92 & $(122.05)$ & 99.13 & $(162.16)$ \\
\hline Total observations & $5,012,128$ & & $1,472,319$ & \\
\hline
\end{tabular}

Note: The Restricted Sample uses data only from 7758 players who play at least 100 matches. The first 3 variables are dummies for when a throw is rock, paper, or scissors (multiplied by 100). The next 3 are the percentages of a player's past throws that were each type, using only the first throw in each match. Variables 7-9 are the same as 4-6, but describing the opponent's history of play instead of the player's. "All throws" are the corresponding percentages for all the opponent's past throws. Opp's Historical Length is the number of previous matches the opponent played. Skew measures the extent to which the opponent's history of first throws deviates from random, $\frac{1}{100} \sum_{i=r, p, s}\left(\% i-\frac{100}{3}\right)^{2}$.

\section{Model}

A standard game of RPS is a simple $3 \times 3$ zero-sum game. The payoffs are shown in Figure 3 . Its only Nash equilibrium is for players to mix $\frac{1}{3}, \frac{1}{3}, \frac{1}{3}$ across rock, paper, and scissors. Because each match is won by the first player to win two throws, and players play multiple matches, the strategies in Roshambull are potentially substantially more complicated: players could condition their play on various aspects of their own or their opponents' histories. A strategy would be a mapping from (1) the match history for the current match so far, (2) one's own history of all matches played, and (3) the space of information one might be shown about one's opponent's history, onto a distribution of throws. 
In addition, Roshambull has a matching process operating in the background, in which players from a large pool are matched into pairs to play a match and then are returned to the pool to be matched again. In the Appendix A.1, we formalize Roshambull in a repeated game framework.

\begin{tabular}{ccccc} 
& & \multicolumn{3}{c}{ Player 2: } \\
& Rock & Rock & Paper & Scissors \\
\cline { 3 - 5 } Player 1: & Paper & $(0,0)$ & $(-1,1)$ & $(1,-1)$ \\
\cline { 3 - 5 } & Scissors & $(1,-1)$ & $(0,0)$ & $(-1,1)$ \\
\cline { 3 - 5 } & & & &
\end{tabular}

Figure 3. Payoffs for a single throw of rock-paper-scissors.

Despite the potential for complexity, we show the equilibrium strategies are still simple.

Proposition 1. In any Nash equilibrium, for every throw of every match, each player correctly expects his opponent to mix $\frac{1}{3}, \frac{1}{3}, \frac{1}{3}$ over rock, paper, and scissors. ${ }^{13}$

Proof. See the Appendix A.1.

The proof shows that since it is a symmetric, zero-sum game, players' continuation values at the end of every match must be zero. Therefore, players are only concerned with winning the match, and not with the effect of their play on their resulting history. We then show that for each throw in the match, if player A correctly believes that player B is not randomizing $\frac{1}{3}, \frac{1}{3}, \frac{1}{3}$, then player A has a profitable deviation.

Same as in the single-shot game, Nash equilibrium implies that players randomize $\frac{1}{3}, \frac{1}{3}, \frac{1}{3}$ both unconditionally and conditional on any information available to their opponent. Out of equilibrium, players may condition their throws on their or their opponents' histories in a myriad of ways. The resulting play may or may not result in an unconditional distribution of play that differs substantially from $\frac{1}{3}, \frac{1}{3}, \frac{1}{3}$. In Section 3, we present evidence that $82 \%$ of experienced players have first throw distributions that do not differ from $\frac{1}{3}, \frac{1}{3}, \frac{1}{3}$, but half respond to their opponents' histories. ${ }^{14}$ While non-random play and responding to information is consistent with Nash beliefs-if the opponent is randomizing $\frac{1}{3}, \frac{1}{3}, \frac{1}{3}$ then any strategy gives a zero expected payoff-it is not consistent with Nash equilibrium because the opponent would exploit that predictability.

\section{Players Respond to Information}

Before examining the data for specific strategies players may be using, we present reduced-form evidence that players respond to the information available to them. To keep the presentation clear and simple, for each analysis we focus on rock, but the results for paper and scissors are analogous, as shown in the Appendix A.2.

We start by examining the dispersion across players in how often they play rock. Figure 4 shows the distribution across experienced players of the fraction of their last 100 throws that are rock. ${ }^{15}$ It also shows the binomial distribution of the fraction of 100 i.i.d. throws that are rock if rock is always played $\frac{1}{3}$ of the time. The distribution from the actual data is substantially more dispersed than the theoretical distribution, suggesting that the fraction of rock played deviates from one third more than one would expect from pure randomness. Doing a chi-squared test on all throws at the player level,

13 Players could use aspects of their history that are not observable to the opponent as a private randomization devices, but conditional on all information available to the opponent, they must be mixing $\frac{1}{3}, \frac{1}{3}, \frac{1}{3}$.

14 We also find serial correlation both across throws within a match and across matches, which is inconsistent with Nash equilibrium.

15 Inexperienced players also have a lot of variance in the fraction of time they play rock, but for them it is hard to differentiate between deviations from $\frac{1}{3}, \frac{1}{3}, \frac{1}{3}$ and noise from randomization. 
we reject ${ }^{16}$ uniform random play for $18 \%$ of experienced players. The rejection rate is lower for less experienced players, but this seems to be due to power more than differences in play. Players who go on to play more games are actually less likely to have their histories deviate significantly from Nash after 20 or 30 games than players who play fewer total games.

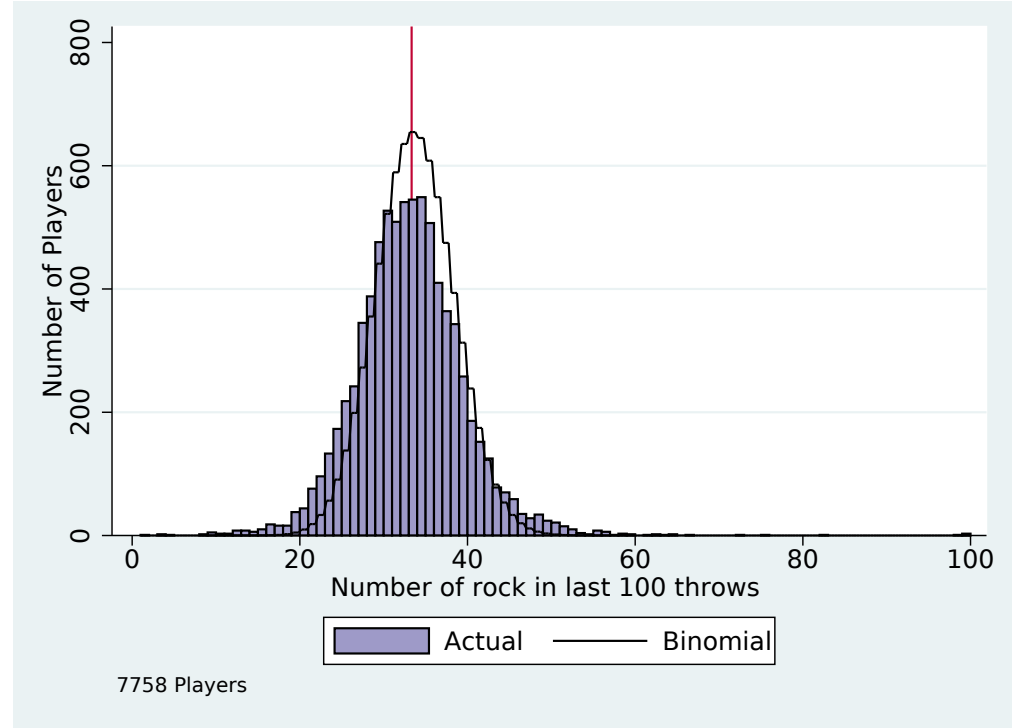

Figure 4. Percent of last 100 Throws that are Rock-Observed and Predicted. Note: For each of the 7758 players with at least 100 matches we calculate the percent of his or her last 100 throws that were rock (purple distribution). We overlay the binomial distribution with $n=100$ and $p=\frac{1}{3}$.

Given this dispersion in the frequency with which players play rock, we test whether players respond to the information they have about their opponent's tendency to play rock-the opponents' historical rock percentage. Table 2 groups throws into bins by the opponents' historical percent rock and reports the fraction of paper, rock, and scissors played. Please note that the percent paper is increasing across the bins and percent scissors is decreasing. Paper goes from less than a third chance to more than a third chance (and scissors goes from more to less) right at the cutoff where rock goes from less often than random to more often than random. ${ }^{17}$ The percent rock a player throws does not vary nearly as much across the bins.

Table 2. Response to Percent Rock.

\begin{tabular}{ccccc}
\hline \multirow{2}{*}{ Opp's Historical \% } & \multicolumn{3}{c}{ Throws (\%) } & \multirow{2}{*}{$N$} \\
\cline { 2 - 4 } & Paper & Rock & Scissors & \\
\hline $0 \%-25 \%$ & 27.07 & 35.84 & 37.09 & $1,006,726$ \\
$25 \%-30 \%$ & 27.11 & 35.23 & 37.66 & 728,408 \\
$30 \%-33 \frac{1}{3} \%$ & 29.87 & 34.72 & 35.41 & 565,623 \\
$33 \frac{1}{3} \%-37 \%$ & 34.21 & 34.44 & 31.35 & 794,886 \\
$37 \%-42 \%$ & 40.45 & 32.58 & 26.97 & 529,710 \\
$42 \%-100 \%$ & 46.58 & 30.35 & 23.06 & $1,056,162$ \\
\hline
\end{tabular}

Note: Matches are binned by the opponent's historical percent of rock on first throws prior to the match. For each range of opponents' historical percent rock, this table reports the distribution of rock, paper, and scissors throws the players use.

16 If all players were playing Nash, we would expect to reject the null for $5 \%$ of players; with $95 \%$ probability we would reject the null for less than $5.44 \%$ of players.

17 If players were truly, strictly maximizing their payoff against the opponent's past distribution, this change would be even more stark, though it would not go exactly from 0 to 1 since the optimal response also depends on the percent of paper (or scissors) played, which the table does not condition on. 
For a more thorough analysis of how this and other information presented to players affects their play, Table 3 presents regression results. The dependent variable is binary, indicating whether a player throws rock. The coefficients all come from one linear probability regression. The first column is the effect for all players, the second column is the additional effect of the covariates for players in the restricted sample; the third column is the additional effect for those players after their first 99 games. For example, a standard deviation increase in the opponent's historical fraction of scissors (0.176) increases the probability that an inexperienced player plays rock by 4.2 percentage points $(100 \times 0.176 \times 0.2376)$; for an experienced player who already played at least 100 games, the increase is 9.4 percentage points $(100 \times 0.176 \times(0.2376+0.1556+0.1381))$.

Table 3. Probability of Playing Rock.

\begin{tabular}{lccc}
\hline Covariate & \multicolumn{2}{c}{ Dependent Var: Dummy for Throwing Rock } \\
\hline & $(1)$ & $(2)$ & $(3)$ \\
Opp's Fraction Paper (first) & $-0.0382^{* * *}$ & $-0.0729^{* * *}$ & $-0.0955^{* * *}$ \\
& $(0.0021)$ & $(0.0056)$ & $(0.0087)$ \\
Opp's Fraction Scissors (first) & $0.2376^{* * *}$ & $0.1556^{* * *}$ & $0.1381^{* * *}$ \\
& $(0.0022)$ & $(0.0060)$ & $(0.0094)$ \\
Opp's Fraction Paper (all) & 0.0011 & $0.0258^{* *}$ & 0.0033 \\
& $(0.0032)$ & $(0.0088)$ & $(0.0138)$ \\
Opp's Fraction Scissors (all) & $0.0416^{* * *}$ & $0.0231^{* *}$ & -0.0208 \\
& $(0.0033)$ & $(0.0093)$ & $(0.0146)$ \\
Opp's Paper Lag & $0.0052^{* * *}$ & -0.0019 & $-0.0043^{*}$ \\
& $(0.0007)$ & $(0.0015)$ & $(0.0019)$ \\
Opp's Scissors Lag & $0.0139^{* * *}$ & $0.0055^{* * *}$ & -0.0017 \\
Own Paper Lag & $(0.0008)$ & $(0.0016)$ & $(0.0020)$ \\
& $-0.0171^{* * *}$ & $0.0239^{* * *}$ & $0.0051^{* *}$ \\
Own Scissors Lag & $(0.0007)$ & $(0.0015)$ & $(0.0019)$ \\
& $-0.0145^{* * *}$ & $0.0208^{* * *}$ & $-0.0047^{*}$ \\
Constant & $(0.0007)$ & $(0.0015)$ & $(0.0019)$ \\
& $0.3548^{* * *}$ & $-0.0264^{* * *}$ & -0.0009 \\
\hline$R^{2}$ & $(0.0006)$ & $(0.0014)$ & $(0.0018)$ \\
$N$ & 0.0172 & & \\
\hline
\end{tabular}

Note: ${ }^{* * *}$, and $* *$ indicate significance at the $10 \%, 5 \%$, and $1 \%$ level respectively. The table shows OLS coefficients from a single regression of a throw being rock on the covariates. The first column is the effect for all players; the second column is the additional effect of the covariates for players in the restricted sample; the third column is the additional effect for those players after their first 100 games. Opp's Fraction Paper (Opp's Fraction Paper (all)) refers to the fraction of the opponent's previous first throws (all throws) that were paper. Opp's Paper Lag (Own Paper Lag) is a dummy for whether the opponent's (player's own) most recent first throw in a match was paper. The Scissor variables are defined analogously for scissors. The regressions also control for the opponent's number of previous matches.

As expected, the effects of the opponent's percent of first throws that were paper is negative and the effect for scissors is positive and both get stronger with experience. ${ }^{18}$ This finding adds to the evidence that experience leads to the adoption of more sophisticated strategies [18,33]. The effect of the opponent's distribution of all throws and the opponent's lagged throws is less clear. ${ }^{19}$ The consistent and strong reactions to the opponent's distribution of first throws motivates our use of that variable in the structural models. If we do the analysis at the player level, the coefficients on opponents' historical distributions are statistically significant for $47 \%$ of experienced players.

18 The Appendix A.2 has the same table adding own history. The coefficients on opponent's history are basically unaffected. The coefficients on own history reflect the imperfect randomization-players who played rock in the past are more likely to play rock.

19 If we run the regression with just the distribution of all throws or just the lags, the signs are as expected, but that seems to be mostly picking up the effect via the opponent's distribution of first throws. 
The fact that players respond to their opponents' histories makes their play somewhat predictable and potentially exploitable. To look at whether opponents exploit this predictability, we first run the regression from Table 3 on half the data and use the coefficients to predict for the other half-based on the opponent's history - the probability of playing rock on each throw. We do the same for paper and scissors. Given the predicted probabilities of play, we calculate the expected payoff to an opponent of playing rock. Table 4 bins throws by the opponents' expected payoff to playing rock and reports the distribution of opponent throws. The probability of playing rock bounces around-if anything, opponents are less likely to play rock when the actual expected payoff is high-the opposite of what we would expect if the predictability of players' throws were effectively exploited.

Table 4. Opponents' Response to Expected Payoff of Rock.

\begin{tabular}{lcccr}
\hline \multirow{2}{*}{ Opponent's Expected Payoff of Rock } & \multicolumn{3}{c}{ Opponent's Throw (\%) } & \multirow{2}{*}{$N$} \\
\cline { 2 - 4 } & Paper & Rock & Scissors & \\
\hline$[-1,-0.666]$ & 30.83 & 40.56 & 28.61 & 2754 \\
{$[-0.666,-0.333]$} & 32.89 & 38.56 & 28.55 & 65,874 \\
{$[-0.333,0]$} & 33.83 & 34 & 32.17 & $1,266,538$ \\
{$[0,0.333]$} & 35.52 & 32.45 & 32.03 & 871,003 \\
{$[0.333,0.666]$} & 34.4 & 34.47 & 31.13 & 12,234 \\
\hline
\end{tabular}

Note: The expected payoff to rock is calculated by running the specification from Table 3 for paper and scissors on half the data, using the coefficients to predict the probability of playing paper minus the probability of playing scissors for each throw in the other half of the sample. This table shows the distribution of opponents' play for different ranges of that expected payoff.

Another way of measuring of the ability to exploit predictability is looking at the win and loss rates. We calculate how often an opponent who responded optimally to the predicted play would win, draw, and lose. We compare these to the rates for the full sample and the experienced sub-sample, keeping in mind that responding to this predicted play optimally would require that the opponent know his own history. Table 5 presents the results. An opponent best responding would win almost $42 \%$ of the time. If players bet $\$ 1$ on each throw, the expected winnings are equal to the probability that they win minus the probability that they lose. The average experienced player would win $1.49 \mathrm{c}$ on the average throw $(34.66 \%$ - loss $22.17 \%=1.49)$, but someone responding optimally to the predictability would win $14.3 c$ on average $(41.66 \%-27.37 \%=14.29)$. (A player playing Nash always breaks even on average.) Though experienced players do better (as previous work has shown (e.g., [18,33])), these numbers indicate that even experienced players are not fully exploiting others' predictability.

Table 5. Win Percentages.

\begin{tabular}{lccccc}
\hline & Wins (\%) & Draws (\%) & Losses (\%) & Wins (\%) - Losses (\%) & $\boldsymbol{N}$ \\
\hline Full Sample & 33.8 & 32.4 & 33.8 & 0 & $5,012,128$ \\
Experienced Sample & 34.66 & 32.17 & 33.17 & 1.49 & $1,472,319$ \\
Best Response to Predicted Play & 41.66 & 30.97 & 27.37 & 14.29 & $2,218,403$ \\
\hline
\end{tabular}

Note: Experienced Sample refers to players who play at least 100 games. "Best Response" is how a player would do if she always played the best response to players' predicted play: the specification from Table 3 (and analogous for paper and scissors) is run on half the data and the coefficients used to predict play for the other half. Wins-Losses shows the expected winnings per throw if players bet $\$ 100$ on a throw.

Since players are responding to their opponent's history, exploiting those responses requires that a player remember her own history of play (since the game does not show one's own history). So, it is perhaps not surprising that players' predictability is not exploited and therefore unsurprising that they react in a predictable manner. Having described in broad terms how players react to the information presented, we turn to existing structural models to test whether play is consistent with these hypothesized non-equilibrium strategies. 


\section{Level- $k$ Behavior}

While level- $k$ theory was developed to analyze single-shot games, it is a useful framework for exploring how players use information about their opponent. The $k_{0}$ strategy is to ignore the information about one's opponent and play a (possibly random) strategy independent of the opponent's history. While much of the existing literature assumes that $k_{0}$ is uniform random, some studies assume that $k_{0}$ players use a salient or focal strategy. In this spirit, we allow players to randomize non-uniformly (imperfectly) when playing $k_{0}$ and assume that the $k_{1}$ strategy best responds to a focal strategy for the opponent- $k_{1}$ players best respond to the opponent's past distribution of first throws. ${ }^{20}$ It seems natural that a $k_{1}$ player who assumes his opponent is non-strategic would use this description of past play as a predictor of future play. ${ }^{21}$ When playing $k_{2}$, players assume that their opponents are playing $k_{1}$ and respond accordingly.

Formal definitions of the different level- $k$ strategies in our context are as follows:

Definition 1. When a player uses a $k_{0}$ strategy in a match, his choice of throw is unaffected by his history or his opponent's history.

We should note that using $k_{0}$ is not necessarily unsophisticated. It could be playing the Nash equilibrium strategy. However, there are two reasons to think that $k_{0}$ might not represent sophisticated play. First, for some players the frequency distribution of their $k_{0}$ play differs significantly from $\frac{1}{3}, \frac{1}{3}, \frac{1}{3}$, suggesting that if they are trying to play Nash, they are not succeeding. Second, more subtly, it is not sophisticated to play the Nash equilibrium if your opponents are failing to play Nash. With most populations who play the beauty contest game, people who play Nash do not win [18]. In RPS, if there is a possibility that one's opponent is playing something other than Nash, there is a strategy that has a positive expected return, whereas Nash always has a zero expected return. (If it turns out the opponent is playing Nash, then every strategy has a zero expected return and so there is little cost to trying something else.) Given that some players differ from $\frac{1}{3}, \frac{1}{3}, \frac{1}{3}$ when playing $k_{0}$ and most do not always play $k_{0}$, Nash is frequently not a best response. ${ }^{22}$

Definition 2. When a player uses the $k_{1}$ strategy in a match, he plays the throw that has the highest expected payoff if his opponent randomizes according to that opponent's own historical distribution of first throws.

We have not specified how a player using $k_{0}$ chooses a throw, but provided the process is not changing over time, his past throw history is a good predictor of play in the current match. To calculate the $k_{1}$ strategy for each throw, we calculate the expected payoff to each of rock, paper, and scissors against a player who randomizes according to the distribution of the opponent's history. The $k_{1}$ strategy is the throw that has the highest expected payoff. (As discussed earlier, it is by definition the same as the strategy that would have been chosen by under fictitious play.) Please note that this is not always the one that beats the opponent's most frequently played historical throw, because it also accounts for the probability of losing (which is worse than a draw). ${ }^{23}$

20 The reduced-form results indicate that players react much more strongly to the distribution of first throws than to the other information provided.

21 Alternatively, a $k_{1}$ player may think that $k_{0}$ is strategic, but playing an unknown strategy so past play is the best predictor of future play.

22 Nash always has an expected payoff of zero. As show in Table 5, best responding can have an expected payoff of $14 \mathrm{c}$ for every dollar bet.

23 Sometimes opponents' distributions are such that there are multiple throws that are tied for the highest expected payoff. For our baseline specification we ignore these throws. As a robustness check we define alternative $k_{1}$-strategies where one throw is randomly chosen to be the $k_{1}$ throw when payoffs are tied or where both throws are considered consistent with $k_{1}$ when payoffs are tied. The results do not change substantially. 
Definition 3. When a player uses the $k_{2}$ strategy in a match, he plays the throw that is the best response if his opponent randomizes uniformly between the throws that maximize the opponent's expected payoff against the player's own historical distribution.

The $k_{2}$ strategy is to play "the best response to the best response" to one's own history. In this particular game $k_{2}$ is in some sense harder than $k_{1}$ because the software shows only one's opponent's history, but players could keep track of their own history.

Both $k_{1}$ and $k_{2}$ depend on the expected payoff to each throw given the assumed beliefs about opponents' play. We calculate the expected payoff by subtracting the probability of losing the throw from the probability of winning the throw, thereby implicitly assuming that players are myopic and ignore the effect of their throw on their continuation value. ${ }^{24}$ This approach is consistent with the literature that analyzes some games as "iterated play of a one-shot game" instead of as an infinitely repeated game [34]. More generally, we think it is a reasonable simplifying assumption. While it is possible one could manipulate one's history to affect future payoffs with an effect large enough to outweigh the effect on this period's payoff, it is hard to imagine how. ${ }^{25}$

Having defined the level- $k$ strategies in our context, we now turn to the data for evidence of level- $k$ play.

\subsection{Reduced-Form Evidence for Level-k Play}

One proxy for $k_{1}$ and $k_{2}$ play is players choosing throws that are consistent with these strategies. Whenever a player plays $k_{1}$ (or $k_{2}$ ) her throw is consistent with that strategy. However, the converse is not true. Players playing the NE strategy of $\frac{1}{3}, \frac{1}{3}, \frac{1}{3}$ would, on average, be consistent with $k_{1}$ a third of the time.

For each player we calculate the fraction of throws that are $k_{1}$-consistent; these fractions are upper bounds on the amount of $k_{1}$ play. No player with more than 20 matches always plays consistent with $k_{1}$. The highest percentage of $k_{1}$-consistent behavior for an individual in our experienced sample is $84 \%$. Figure 5 a shows the distribution of the fraction of $k_{1}$-consistency across players. It suggests that at least some players use $k_{1}$ at least some of the time: the distribution is to the right of the vertical $\frac{1}{3}$-line and there is a substantial right tail. To complement the graphical evidence, we formally test whether the observed frequency of $k_{1}$-consistent play is significantly greater than expected under random play. For each player with at least 100 games, we calculate the probability of observing at least as many throws consistent with $k_{1}$ if the probability of a given throw being $k_{1}$-consistent were only $1 / 3$. The probability is less than $5 \%$ for $47 \%$ of players.

Given that players seem to play $k_{1}$ some of the time, players could benefit from playing $k_{2}$. Figure $5 \mathrm{~b}$ shows the distribution of the fraction of actual throws that are $k_{2}$-consistent. The observed frequency of $k_{2}$ play is slightly to the left of that expected with random play, but we cannot reject random play for a significant number of players. This lack of evidence for $k_{2}$ play is perhaps unsurprising given that players are not shown the necessary information.

24 In the proof of Proposition 1 we show that in Nash equilibrium, histories do not affect continuation values, so in equilibrium it is a result, not an assumption, that players are myopic. However, out of Nash equilibrium, it is possible that what players throw now can affect their probability of winning later rounds.

25 One statistic that we thought might affect continuation values is the skew of a player's historical distribution. As a player's history departs further from random play, there is more opportunity for opponent response and player exploitation of opponent response. We ran multinomial logits for each experienced player on the effect of own history skewness on the probability of winning, losing, or drawing. The coefficients were significant for less than (the expected false positives of) $5 \%$ of players. This provides some support to our assumption that continuation values are not a primary concern. 


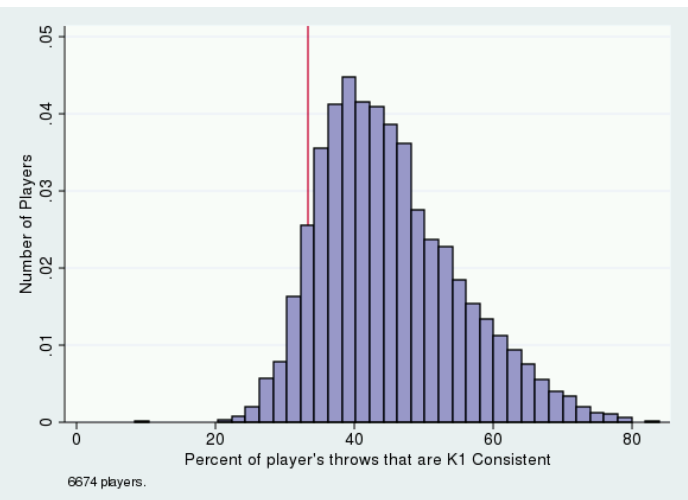

(a)

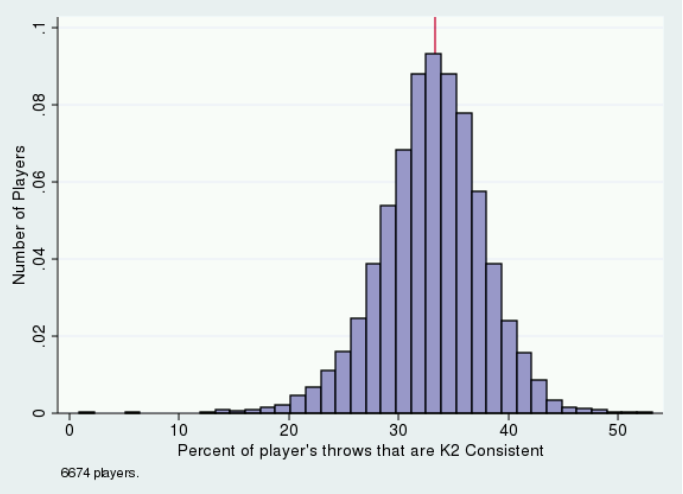

(b)

Figure 5. Level- $k$ consistency. Note: These graphs show the distribution across the 6674 players who have 100 games with uniquely defined $k_{1}$ and $k_{2}$ strategies of the fraction of throws that are $k_{1}$ - and $k_{2}$-consistent. The vertical line indicates $\frac{1}{3}$, which we would expect to be the mean of the distribution if throws were random. (a) Percent of player's throws that are $k_{1}$-consistent. (b) Percent of player's throws that are $k_{2}$-consistent.

If we assume that players use either $k_{0}, k_{1}$, or $k_{2}$ then we can get a lower bound on the amount of $k_{0}$. For each player we calculate the percentage of throws that are consistent with neither $k_{1}$ nor $k_{2}$. We do not expect this bound to be tight because, in expectation, a randomly chosen $k_{0}$ play will be consistent with either the $k_{1}$ or $k_{2}$ strategy about $\frac{1}{3}+\left(1-\frac{1}{3}\right) \times \frac{1}{3} \approx 0.56$ of the time. The mean lower bound across players with at least 100 matches is $37 \%$. The minimum is $8.2 \%$ and the maximum is $77 \%$.

The players do have an incentive to use these strategies. Averaging across the whole dataset, always playing $k_{1}$ would allow a player to win $35.09 \%$ (and lose $32.61 \%$ ) of the time. If a player always played $k_{2}$ he would win $42.68 \%$ (and lose $27.74 \%$ ) of the time. While these numbers may be surprising, if an opponent plays $k_{1}$ just $14 \%$ of the time and plays randomly the rest of the time, the expected win rate from always play $k_{2}$ would be $0.14 \times 1+0.86 \times 0.33=0.426$. It seems that memory or informational constraints prevent players from employing what would be a very effective strategy.

\section{Multinomial Logit}

Before turning to the structural model, we can use a multinomial logit model to explore whether a throw being $k_{1}$-consistent increases the probability that a player chooses that throw. For each player, we estimate a multinomial logit where the utilities are

$$
U_{i}^{j}=\alpha^{j}+\beta \cdot 1\left\{k_{1, i}=j\right\}+\varepsilon_{i}^{j}
$$

where $j=r, p, s$ and $1\left\{k_{1, i}=i\right\}$ is an indicator for when $j$ is the $k_{1}$-consistent action for throw $i$. Figure 6 shows the distribution of $\beta$ s across players. The mean is 0.52 .

The marginal effect varies slightly with the baseline probabilities, $\frac{\partial \operatorname{Pr}\{i\}}{\partial x_{i}}=\beta \times \operatorname{Pr}\{i\}(1-\operatorname{Pr}\{i\})$, but is approximately $\frac{1}{3}\left(1-\frac{1}{3}\right)=\frac{2}{9}$ times the coefficient. Hence, on average, a throw being $k_{1}$-consistent means it is 12 percentage points more likely to be played. Given that the standard deviation across experienced players in the percent of rock, paper, or scissors throws is about 5 percentage points, this is a large average effect. The individual-level coefficient is positive and significant for $64 \%$ of players. 


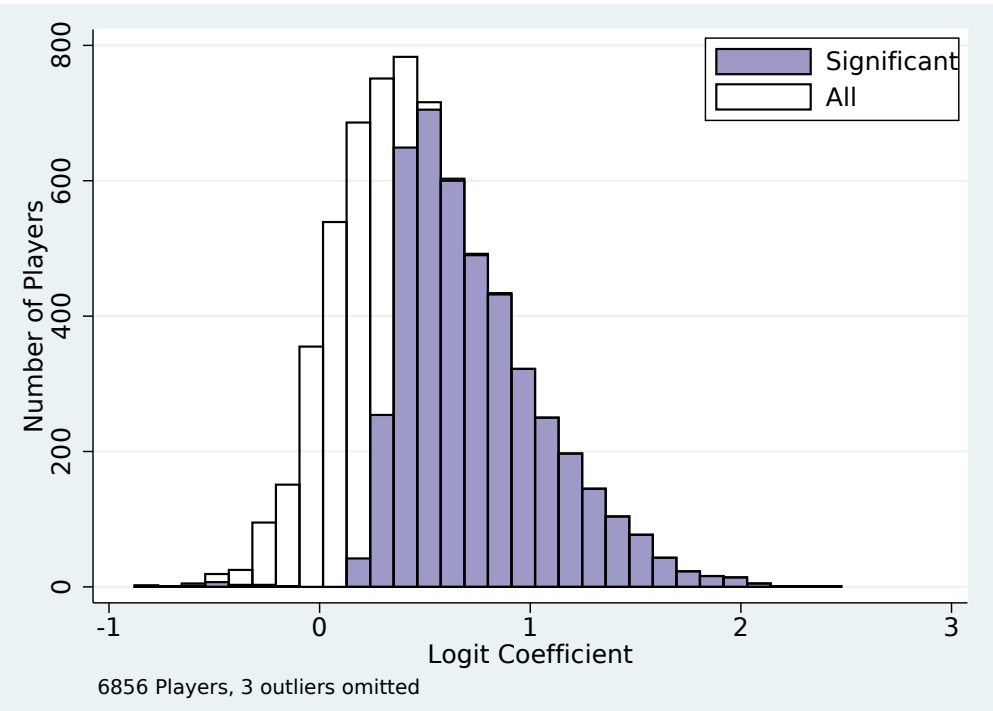

Figure 6. Coefficient in the $k_{1}$ multinomial logit. Note: The coefficient is $\beta$ from the logit estimation, run separately for each player, $U_{i}^{\text {rock }}=\alpha^{\text {rock }}+\beta \cdot k_{1, i}^{\text {rock }}+\epsilon_{i}^{\text {rock }}$, analogously for paper and scissors, where $k_{1}^{\text {rock }}$ is a dummy for whether rock is the $k_{1}$-consistent thing to do on throw $i$. Outliers more than 4 standard deviations from the mean are omitted.

\subsection{Maximum Likelihood Estimation of a Structural Model of Level-k Thinking}

The results presented in the previous sections provide some evidence as to what strategies are being employed by the players in our sample, but they do not allow us to identify with precision the frequency with which strategies are employed-we can say that throws are $k_{1}$ more often than would happen by chance, but cannot estimate what fraction of the time a player is playing a throw because it is $k_{1}$. To obtain point estimates of each player's proportion of play by level- $k$, along with standard errors, we need additional assumptions.

Assumption 1. All players use only the $k_{0}, k_{1}$, or $k_{2}$ strategies in choosing their actions.

Assumption 1 restricts the strategy space, ruling out any approach other than level- $k$, and restricting players not to use levels higher than $k_{2}$. We limit our modeling to levels $k_{2}$ and below, both for mathematical simplicity and because there is little reason to believe that higher levels of play are commonplace, both based on the low rates of $k_{2}$ play in our data, and rarity of $k_{3}$ and higher play in past experiments. ${ }^{26}$

Assumption 2. Whether players choose to play $k_{0}, k_{1}$, or $k_{2}$ on a given throw is independent of which throw (rock, paper, or scissors) each of the strategies would have them play.

Assumption 2 implies, for example, that the likelihood that a player chooses to play $k_{2}$ will not depend on whether it turns out that the $k_{2}$ action is rock or is paper. This independence is critical to the conclusions that follow. Please note that Assumption 2 does not require that a player commit to having the same probabilities of using $k_{0}, k_{1}$, and $k_{2}$ strategies across different throws.

26 As an aside, in the case of RPS the level $k_{j+6}$ strategy is identical to the level $k_{j}$ strategy for $j \geq 1$, so it is impossible to identify levels higher than 6 . One might expect $k_{j}$ to be equivalent to $k_{j+3}$, but $k_{1}, k_{3}$ and $k_{5}$ strategies depend on the opponent's history, with one being rock, one being paper, and one being scissors, while levels $k_{2}, k_{4}$ and $k_{6}$ strategies depend on one's own history. So, with many games all strategies $k_{j}$ with $j<7$ are separately identified. This also implies that the $k_{1}$ play we observe could in fact be $k_{7}$ play, but we view this as highly unlikely. 
Given these assumptions, we can calculate the likelihood of observing a given throw as a function of five parameters: the probability of using the $k_{0}$-strategy and choosing a given throw $\left(\hat{k}_{0}^{r}, \hat{k}_{0}^{p}, \hat{k}_{0}^{s}\right)$ and the probability of using the $k_{1}$ and $k_{2}$ strategies $\left(\hat{k}_{1}, \hat{k}_{2}\right)$. The probability of observing a given throw $i$ is

$$
\hat{k}_{1} \cdot 1\left\{k_{1}=i\right\}+\hat{k}_{2} \cdot 1\left\{k_{2}=i\right\}+\hat{k}_{0}^{i}
$$

where $1\{\cdot\}$ is an indicator function, equal to one when the statement in braces is true and zero otherwise. This reflects the fact that the throw will be $i$ if the player plays $k_{1}$ and the $k_{1}$ strategy says to play $i\left(\hat{k}_{1} \cdot 1\left\{k_{1}=i\right\}\right)$ or the player plays $k_{2}$ and the $k_{2}$ strategy says to play $i\left(\hat{k}_{2} \cdot 1\left\{k_{2}=i\right\}\right)$ or the player plays $k_{0}$ and chooses $i\left(\hat{k}_{0}^{i}\right)$. Table 6 summarized the parameters; the probabilities sum to one, $\hat{k}_{1}+\hat{k}_{2}+\hat{k}_{0}^{r}+\hat{k}_{0}^{p}+\hat{k}_{0}^{s}=1$, so there are only 4 independent parameters.

Table 6. Parameters of the Structural Model.

\begin{tabular}{ll}
\hline Variable & Definition \\
\hline$\hat{k}_{0}^{r}$ & fraction of the time a player plays $k_{0}$ and chooses rock \\
$\hat{k}_{0}^{p}$ & fraction of the time a player plays $k_{0}$ and chooses paper \\
$\hat{k}_{0}^{s}$ & fraction of the time a player plays $k_{0}$ and chooses scissors \\
$\hat{k}_{1}$ & fraction of the time a player plays $k_{1}$ \\
$\left(\hat{k}_{2}\right)$ & $1-\hat{k}_{1}-\hat{k}_{0}^{r}-\hat{k}_{0}^{p}-\hat{k}_{0}^{s}$ (not an independent parameter) \\
\hline
\end{tabular}

Note: $\hat{k}_{0}^{r}$ is not equal to the fraction of $k_{0}$ throws that are rock; that conditional probability is given by $\frac{\hat{k}_{0}^{r}}{\hat{k}_{0}^{r}+\hat{k}_{0}^{p}+\hat{k}_{0}^{s}}$.

For each player, the overall log-likelihood depends on 12 statistics from the data. For each throw type $(i=R, P, S)$, let $n_{12}^{i}$ be the number of throws of type $i$ that are consistent with $k_{1}$ and $k_{2}, n_{1}^{i}$ the number of throws of type $i$ consistent with just $k_{1}, n_{2}^{i}$ the number of throws of type $i$ consistent with just $k_{2}$, and $n_{0}^{i}$ the number of throws of type $i$ consistent with neither $k_{1}$ nor $k_{2}$. Given these statistics, the log-likelihood function is

$$
\mathcal{L}\left(\hat{k}_{1}, \hat{k}_{2}, \hat{k}_{0}^{r}, \hat{k}_{0}^{p}, \hat{k}_{0}^{s}\right)=\sum_{i=r, p, s}\left(n_{12}^{i} \ln \left(\hat{k}_{1}+\hat{k}_{2}+\hat{k}_{0}^{i}\right)+n_{1}^{i} \ln \left(\hat{k}_{1}+\hat{k}_{0}^{i}\right)+n_{2}^{i} \ln \left(\hat{k}_{2}+\hat{k}_{0}^{i}\right)+n_{0}^{i} \ln \left(\hat{k}_{0}^{i}\right)\right) .
$$

For each experienced player we use maximum likelihood to estimate $\left(\hat{k}_{1}, \hat{k}_{2}, \hat{k}_{0}^{r}, \hat{k}_{0}^{p}, \hat{k}_{0}^{s}\right) .{ }^{27}$ Given the estimates, standard errors are calculated analytically. ${ }^{28}$

This approach allows us to not count as $k_{1}$ those throws that are likely $k_{0}$ or $k_{2}$ and only coincidentally consistent with $k_{1}$; it is more sophisticated than simply looking at the difference between the rate of $k_{1}$-consistency and $1 / 3$ as we do in Figure 5 a. If a player is biased towards playing rock and rock is the $k_{1}$ move in disproportionately many of their matches, we would not want to count those plays as $k_{1}$. Conversely, if a player always played $k_{1}$, we would not want to say that $1 / 3$ of those were due to chance. Essentially, the percentage of the time a player uses the $k_{1}$ strategy is estimated from the extent to which that player is more likely to play rock (or paper or scissors) when it is $k_{1}$-consistent than when it is not $k_{1}$-consistent.

Table 7 summarizes the estimates of $k_{0}, k_{1}$, and $k_{2}$ : the average player uses $k_{0}$ for $73.8 \%$ of throws, $k_{1}$ for $18.5 \%$ of throws and $k_{2}$ for $7.7 \%$ of throws. Weighting by the precision of the estimates or by the number of games does not change these results substantially. As the minimums and maximums

27 Since we do the analysis within player, the estimates would be very imprecise for players with fewer games.

28 We derive the Hessian of the likelihood function, plug in the estimates, and take the inverse. 
suggest, these averages are not the result of some people always playing $k_{1}$ while others always play $k_{2}$ or $k_{0}$. Most players mix, using a combination of mainly $k_{0}$ and $k_{1} \cdot{ }^{29}$

Table 7. Summary of $k_{0}, k_{1}$, and $k_{2}$ estimates.

\begin{tabular}{lcccccc}
\hline Variable & Mean & SD & Median & Min & Max \\
\hline$k_{0}$ & 0.738 & 0.16 & 0.75 & 0.19 & 1.00 \\
$k_{1}$ & 0.185 & 0.14 & 0.16 & 0.00 & 0.77 \\
$k_{2}$ & 0.077 & 0.08 & 0.06 & 0.00 & 0.41 \\
\hline$N=6639$ & & & & & \\
\hline
\end{tabular}

Note: Based on the 6639 players with 100 clean matches with well-defined $k_{1}$ and $k_{2}$ strategies.

Table 8 reports the share of players for whom we can reject with $95 \%$ confidence their never playing a particular level- $k$ strategy. Using standard errors calculated separately for each player from the Hessian of the likelihood function, we test whether 0 or 1 fall within the $95 \%$ confidence intervals of $\hat{k}_{1}, \hat{k}_{2}$, and $1-\hat{k}_{1}-\hat{k}_{2}$. Almost all players (93\%) appear to use $k_{0}$ at some point. About $63 \%$ of players use $k_{1}$ at some stage, but we can reject exclusive use of $k_{1}$ for all but two out of 6389 players. Finally, for only about 12 percent of players do we have significant evidence that they use $k_{2}$.

Table 8. Percent of players we reject always or never playing a strategy.

\begin{tabular}{lccc}
\hline Variable & $\begin{array}{c}\text { 95\% CI Does Not } \\
\text { Include 0 }\end{array}$ & $\begin{array}{c}\text { 95\% CI Does Not } \\
\text { Include 1 }\end{array}$ & $\begin{array}{c}\text { 95\% CI Does Not } \\
\text { Include 0 or 1 }\end{array}$ \\
\hline$k_{0}$ & $93.11 \%$ & $58.30 \%$ & $57.51 \%$ \\
$k_{1}$ & $62.87 \%$ & $99.97 \%$ & $62.87 \%$ \\
$k_{2}$ & $11.54 \%$ & $95.57 \%$ & $11.54 \%$ \\
\hline$N=6389$ & & &
\end{tabular}

Note: All percentages refer to the 6389 players who have 100 matches where the $k_{1}$ and $k_{2}$ strategies are well-defined and for whom we can calculate standard errors on the estimates using the Hessian of the likelihood function.

For each player, we can also examine the estimated fraction of rock, paper, and scissors when they play $k_{0}$. The distribution differs significantly from random uniform for 1252 players $(20 \%)$. This is similar to the number of players whose raw throw distributions differ significantly from uniform $(18 \%)$, suggesting that the deviations from uniform are not due to players playing $k_{1}$ and the distribution of the indicated $k_{1}$ play deviating significantly from uniform.

For this analysis we made structural assumptions that are specific to our setting and use maximum likelihood estimation to identify player strategies given that structure. This is a similar approach to papers that identify player strategies in other settings. Kline [36] presents a method for identification under a continuous action space and applies this method to two-person guessing games. Hahn et al. [37] present a method for a setting with a continuous action space where the parameters of the game evolve over time. They can identify player strategies for a p-beauty contest (where the goal of the game is to guess the value $p$ times the average of all the guesses) by checking that a player behaves consistently with a strategy given the changing values of $p<1$. The main difference is that in our context, the action space over which players randomize is discrete. Houser et al. [38] presents a method for a dynamic setting with discrete action where different player type beliefs cause them to perceive the continuation value of the actions differently, given the same game state. In our setting, we do not find evidence that players are to a significant extent choosing actions to manipulate their histories and hence maximize a continuation value, so we are able to use a simpler framework.

29 Other work, such as [35], has found evidence of players mixing levels of sophistication across different games. 


\subsection{Cognitive Hierarchy}

The idea that players might use a distribution over the level- $k$ strategies naturally connects to the cognitive hierarchy model of Camerer et al. [39]. They also model players as having different levels of reasoning, but the higher types are more sophisticated than in level- $k$. Levels 0 and 1 of the cognitive hierarchy strategies are the same as in the level- $k$ model; level 2 assumes that other players are playing either level- 0 or level-1, in proportion to their actual use in the population, and best responds to that mixture. To test if this more sophisticated version of two levels of reasoning fits the data better, we do another maximum likelihood estimation. Since we again limit to two iterations of reasoning, this is a very restricted version of cognitive hierarchy.

The definitions of $c h_{0}$ and $c h_{1}$ are the same as $k_{0}$ and $k_{1}$.

Definition 4. When a player uses the $\mathrm{ch}_{2}$ strategy in a match, he plays the throw that is the best response if the opponent

- $\quad$ randomizes according to the opponent's historical distribution $79.92 \%$ of the time

- chooses (randomly between) the throw(s) that maximize expected payoff against the player's own historical distribution $20.08 \%$ of the time

The percentages come from observed frequencies in the level- $k$ estimation. When players play either $k_{0}$ or $k_{1}$, they play $k_{0} \frac{73.80}{73.80+18.54}=79.92 \%$ of the time..$^{30}$ Analogous to Assumptions 1 and 2 above, we assume that players use only $c h_{0}, c h_{1}$ and $c h_{2}$, and that which strategy they choose is independent of what throw the strategy dictates.

Table 9 summarizes the estimates: the average player uses $c h_{0}$ for $75.0 \%$ of throws, $c h_{1}$ for $16.1 \%$ of throws, and $c h_{2}$ for $9.0 \%$ of throws. Weighting by the precision of the estimates or by the number of games a player plays does not change these substantially. These results are similar to what we found for level- $k$ strategies; this suggests that the low rates we found of $k_{2}$ were not a result of restricting $k_{2}$ to respond only to $k_{1}$ and ignore the prevalence of $k_{0}$ play.

Table 9. Summary of $c h_{0}, c h_{1}$, and $c h_{2}$ estimates.

\begin{tabular}{|c|c|c|c|c|c|}
\hline Variable & Mean & SD & Median & Min & Max \\
\hline$k_{0}$ & 0.750 & 0.16 & 0.77 & 0.17 & 1.00 \\
\hline$k_{1}$ & 0.161 & 0.14 & 0.14 & 0.00 & 0.77 \\
\hline$k_{2}$ & 0.089 & 0.07 & 0.08 & 0.00 & 0.49 \\
\hline \multicolumn{6}{|l|}{$N=6856$} \\
\hline
\end{tabular}

\subsection{Naive Level-k Strategies}

Even if a player expects his opponent to play as she did in the past, he may not calculate the expected return to each strategy. Instead he may employ the simpler strategy of playing the throw that beats the opponent's most common historical throw. Put another way, he may only consider maximizing his probability of winning instead of weighing it against the probability of losing as is done in an expected payoff calculation. We consider this play naive and define alternative versions of $k_{1}$ and $k_{2}$ accordingly.

Definition 5. When a player uses the naive $k_{1}$ strategy in a match, he plays the throw that will beat the throw that his opponent has played most frequently in the past.

30 To fully calculate the equilibrium, we could repeat the analysis using the frequencies of $c h_{0}$ and $c h_{1}$ found below and continue until the frequencies converged, but since the estimated $c h_{0}$ and $c h_{1}$ are near the $79 \%$ and $20 \%$ we started with, we do not think this computationally intense exercise would substantially change the results. 
Definition 6. When a player uses the naive $k_{2}$ strategy in a match and has played throw $i$ most frequently in the past, then he plays the throw that beats the throw that beats $i$.

Table 10 summarizes the estimates for naive play. The average player uses $k_{0}$ for $72.2 \%$ of throws, naive $k_{1}$ strategy for $21.1 \%$ of throws and naive $k_{2}$ strategy for $6.7 \%$ of throws. As before, weighting by the precision of the estimates or by the number of games a player plays does not change these results substantially. Most players use a mixed strategy, mixing primarily over $k_{0}$ and naive $k_{1}$ strategy.

Table 10. Summary of Naive $k_{0}, k_{1}$, and $k_{2}$ estimates.

\begin{tabular}{lcccccc}
\hline Variable & Mean & SD & Median & Min & Max \\
\hline$k_{0}$ & 0.722 & 0.18 & 0.75 & 0.07 & 1.00 \\
$k_{1}$ & 0.211 & 0.17 & 0.18 & 0.00 & 0.93 \\
$k_{2}$ & 0.067 & 0.08 & 0.04 & 0.00 & 0.46 \\
\hline$N=5692$ & & & & & \\
\hline
\end{tabular}

Note: Based on the $\overline{5692 \text { players with } 100 \text { clean matches with well-defined naive }} k_{1}$ and $k_{2}$ strategies.

The opposite naive strategy would be for players to minimize their probability of losing, playing the throw that is least likely to be beat. Running the same model for that strategy we find almost no evidence of $k_{1}$ or $k_{2}$ play, suggesting that players are more focused on the probability of winning. This is consistent with the reduced-form evidence that the effect on the probability of the opponent's fraction of past scissors played is about eight times as large as the effect of the opponent's fraction of past paper played.

\subsection{Comparisons}

Since the fraction of each strategy players use is not a good indication of the overall fit of the model, we do a likelihood comparison test of the three models-baseline level- $k$, cognitive hierarchy, and naive level- $k$ - for each player. If $l l_{j}$ is the log-likelihood of model $j$ then

$$
\operatorname{Prob}_{j}=\frac{\exp \left(l l_{j}\right)}{\exp \left(l l_{k}\right)+\exp \left(l l_{c h}\right)+\exp \left(l l_{\text {naive }}\right)}
$$

is the probability that the data was generated by model $j$, assuming it was generated by one of the three models. Using the likelihoods based only on throws for which all three strategies are uniquely defined, Table 11 reports the number of players for whom each model has the highest probability. We look both at all 5405 players that have 100 such throws and the subset of players for whom the most likely model has a probability over 95\%. Among all players, naive level- $k$ is the most common $(50 \%)$ and cognitive hierarchy (35\%) is more common than level- $k(15 \%)$. The difference is even starker among the players who strongly favor one model: naive level- $k$ is the best fit for $85 \%$ of such players. It seems that many players appear to be playing traditional $k_{1}$ mainly because it frequently indicates the same throw as the naive $k_{1}$ strategy.

The game and context is different, but all three of these related models suggest that players of Roshambull use considerably fewer levels of iteration in their reasoning process compared to participants in other games and other experiments. Bosch-Domenech et al. [25] found that less than a fourth of the players who used the $k$-strategies we discuss in this paper were $k_{0}$ players. Whereas we found that on average players used zero iterations of reasoning between $72 \%$ and $75 \%$ of the time. Camerer et al. [39] suggest that players iterate 1.5 steps on average in many games. In comparison, in our level- $k$ model we find that our average player uses $1 \times 0.185+2 \times .077=0.339$ levels of iterated 
rationality. ${ }^{31}$ Stahl and Wilson [16] reported that an insignificant fraction of players was $k_{0}, 24 \%$ were $k_{1}$ players, $49 \%$ were $k_{2}$ players, and the remaining $27 \%$ were "Nash types." In contrast, we found that the majority of plays were $k_{0}\left(c h_{0}\right)$ and that $k_{1}\left(c h_{1}\right)$ outnumbered $k_{2}\left(c h_{2}\right)$, though in this game $k_{0}$ is closer to Nash than either $k_{1}$ or $k_{2}$.

Table 11. Likeliest Model by Player.

\begin{tabular}{lccc}
\hline & \multicolumn{3}{c}{ Model } \\
\cline { 2 - 4 } & Level- $\boldsymbol{k}$ & Cognitive Hierarchy & Naive \\
\hline All Players & 798 & 1833 & 2629 \\
& $14.8 \%$ & $33.9 \%$ & $48.6 \%$ \\
\hline Players $>95 \%$ Prob & 13 & 188 & 1148 \\
& $1.0 \%$ & $13.9 \%$ & $85.1 \%$ \\
\hline
\end{tabular}

Note: Based on the 5405 players that have 100 games where the strategy under all three models-baseline level- $k$, cognitive hierarchy, and naive level- $k$ - is uniquely defined, this table shows the number and percent of players for whom each model has the highest log-likelihood. The bottom panel shows only the players for whom the posterior probability—as formulated in Equation (1)—of the most likely model is greater than $95 \%$.

One explanation for the differences between our results and the past literature is that most of the players do not deviate substantially from equilibrium play, making the expected payoffs to $k_{1}$ relatively small. Also, the setup of RPS does not suggest a level- $k$ thinking mindset as strongly as the p-beauty contest games or other games specifically designed to measure level- $k$ behavior. Our more flexible definition of $k_{0}$ play may also explain its higher estimate. The dearth of $k_{2}$ play is especially striking in our context given the high returns to playing $k_{2}$. This is likely a result of the Facebook application not showing players their histories, so players had to keep track of that on their own to effectively play $k_{2}$.

Another explanation is that we restrict the strategy space, excluding both Nash equilibrium and alternative ways in which the players could react to their opponent's information. It seems players respond more to the first throw history than other information, but there may be other strategies that combine pieces of information in ways which we do not model. Bosch-Domenech et al. [25], for example, considered equilibrium, fixed point, degenerate, and non-degenerate variants of iterated best response, iterated dominance, and even "experimenter" strategies. Not all of these translate into the RPS setup, but any strategies that our model left out might look like $k_{0}$ play when the strategy space is restricted.

\subsection{When Are Players' Throws Consistent with $k_{1}$ ?}

Though we find relatively low levels of $k_{1}$ play, we do find some; the result that many of the players seem to be mixing strategies raises the question of when they choose to play $k_{0}, k_{1}$, and $k_{2}$. Our structural model assumes that the strategy players choose is independent of the throw dictated by each of the strategies. It does not require that which strategy they choose be independent of the expected payoffs, but the maximum likelihood estimation (MLE) model cannot give us insight into how expected payoffs may affect play. This is partially because the MLE model does not allow us to categorize individual throws as following a specific strategy.

To try to get at when players use $k_{1}$, we return to using $k_{1}$-consistency as a proxy for possible $k_{1}$ play. We test two hypotheses. First, the higher the expected payoff to playing $k_{1}$, the more likely a player is to play $k_{1}$. For example, the expected return to playing $k_{1}$, relative to playing randomly, is much higher if the opponent's history (or expected distribution) is $40 \%$ rock, $40 \%$ paper, $20 \%$ scissors than if it is $34 \%$ rock, $34 \%$ paper, $32 \%$ scissors. Also, a high $k_{1}$ payoff may indicate that the opponent is unlikely to play a lot of $k_{2}$ (which leads to mean reversion), which increases the expected return to $k_{1}$.

31 Wright and Leyton-Brown [40]'s estimates are closer to ours, with 95\% confidence interval for the average number of iterative steps of $[0.51,0.59]$. 
The second hypothesis is that a player will react more to a higher $k_{1}$ payoff when his opponent has played more games. A $40 \%$ rock, $40 \%$ paper, $20 \%$ scissors history is more informative if it is based on 100 past throws than if it is based on only 10 throws. ${ }^{32}$ We bin opponent history length into terciles and interact the dummy variables for the middle and top tercile with the $k_{1}$ payoff. We also analyze whether these effects vary by player experience; we interact all the covariates with whether a player is in the restricted sample (they eventually play $\geq 100$ matches) and whether they have played 100 matches before the current match.

Table 12 presents empirical results from testing these hypotheses. ${ }^{33}$ The $k_{1}$ payoff is the expected payoff to playing $k_{1}$ assuming the opponent randomizes according to his history. Its standard deviation is 0.23 . Using coefficients from Column 3 , we see that for inexperienced players a one-standard-deviation increase in payoff to the $k_{1}$ strategy, increases the probability the throw is $k_{1}$-consistent by 1.6 percentage points $(0.23 \cdot 0.071 \approx 1.6 \%)$ when opponents have played fewer than 14 games, 9 percentage points $(0.23 \cdot(0.071+0.318) \approx 9 \%)$ when opponents have a medium history, and 14 percentage points $(0.23 \cdot(0.071+0.528) \approx 14 \%)$ when opponents have played over 46 games. Given that $43 \%$ of all throws are $k_{1}$-consistent, these latter two effects are substantial. Experienced players react slightly less to the $k_{1}$-payoff when opponents have short histories, but their reactions to opponents with medium or long histories are somewhat larger.

Table 12. Effect of Expected $k_{1}$ Payoff on $k_{1}$-Consistency (OLS).

\begin{tabular}{lccc}
\hline & $\mathbf{( 1 )}$ & $\mathbf{( 2 )}$ & $\mathbf{( 3 )}$ \\
\hline K1 Payoff & $0.068^{* * *}$ & $0.105^{* * *}$ & $0.071^{* * *}$ \\
& $(0.0012)$ & $(0.0013)$ & $(0.0016)$ \\
High Opponent Experience & & & $-0.076^{* * *}$ \\
& & & $(0.0017)$ \\
Medium Opponent Experience & & $-0.055^{* * *}$ \\
& & & $(0.0015)$ \\
K1 Payoff X High Opponent Experience & & & $0.528^{* * *}$ \\
& & & $(0.010)$ \\
K1 Payoff X Medium Opponent Experience & & $0.318^{* * *}$ \\
& $0.015^{* * *}$ & & $(0.0050)$ \\
Experienced & $(0.0015)$ & & \\
& $0.068^{* * *}$ & $0.041^{* * *}$ & $-0.021^{* * *}$ \\
Exp X Payoff from Playing K1 & $(0.0039)$ & $(0.0038)$ & $(0.0046)$ \\
& & & $-0.019^{* * *}$ \\
Exp X High Opponent Experience & & & $(0.0034)$ \\
& & & $\left(0.023^{* * *}\right.$ \\
Exp X Medium Opponent Experience & & & $0.091^{* * *}$ \\
Exp X K1 Payoff X High Opponent Experience & & & $(0.021)$ \\
& & & $0.095^{* * *}$ \\
Exp X K1 Payoff X Medium Opponent Experience & & & $(0.013)$ \\
Own Games>100 & & & \\
Own Games>100 X Payoff from Playing K1 & $(0.0022)$ & & $0.021^{* * * *}$ \\
& $0.082^{* * *}$ & $0.072^{* * *}$ & $(0.0049)$ \\
\hline
\end{tabular}

32 Similar predictions could be made about $k_{2}$ play; however, since we find that $k_{2}$ is used so little, we do not model $k_{2}$ play in this section. See Appendix A.2

33 We present OLS coefficients instead of logit, so they will be comparable when we add fixed-effects, which do not work with logit. When running logits at the player level, the median coefficients are in the range of the above coefficients, but the model is totally lacking power-the coefficients are significant for less than $5 \%$ of players. 
Table 12. Cont.

\begin{tabular}{|c|c|c|c|}
\hline & (1) & (2) & (3) \\
\hline Own Games > 100 X High Opponent Experience & & & $\begin{array}{l}0.012 * * * \\
(0.0026)\end{array}$ \\
\hline Own Games $>100$ X Medium Opponent Experience & & & $\begin{array}{c}0.022 * * * \\
(0.0035)\end{array}$ \\
\hline Own Games>100 X K1 Payoff X High Opponent Experience & & & $\begin{array}{l}-0.025 \\
(0.023)\end{array}$ \\
\hline Own Games >100 X K1 Payoff X Medium Opponent Experience & & & $\begin{array}{c}0.014 \\
(0.016)\end{array}$ \\
\hline Player Fixed Effects & No & Yes & Yes \\
\hline Observations & $4,130,024$ & $4,130,024$ & $4,130,024$ \\
\hline Adjusted $R^{2}$ & 0.003 & 0.004 & 0.008 \\
\hline
\end{tabular}

Note: ${ }^{* * *}$ indicates significance at the $1 \%$ level. S.E.'s are clustered by player. The dependent variable is a dummy for a throw being $k_{1}$-consistent. ' $k_{1}$ Payoff' is the expected payoff to playing $k_{1}$ if the opponent randomizes according to his history (ranges from 0 to 1 ). 'High opp exp' is a dummy for opponents who have 47 or more past games; 'Medium opp exp' is a dummy for opponents with 14 to 46 past games. 'Experienced' is a dummy for players who eventually play at $\geq 100$ games. 'Own Games $>100$ ' indicates the player has already played at least 100 games. The ' $X$ ' indicates the interaction between the dummies and other covariates.

While we expect the correlation between opponent's history length and playing $k_{1}$ to be negative-since longer histories are less likely to show substantial deviation from random-we do not have a good explanation for why the direct effect of opponent's history length is negative, even when controlling for the $k_{1}$ payoff. Perhaps the players are more wary of trying to exploit a more experienced player.

\section{5. (Non-Equilibrium) Quantal Response}

The above evidence that $k_{1}$-consistent play is more likely when the expected payoff is higher, naturally leads us to a model of play that is more continuous. In some sense level- $k$ strategies are all or nothing. If a throw has the highest expected payoff against the opponent's historical distribution, then the $k_{1}$ strategy says to play it, even if expected payoff is very small. A related, but different strategy is for players to choose each throw with a probability that is increasing in its expected payoff against the opponent's historical distribution of play. This is related to the idea behind quantal response equilibrium [30], but replacing the requirement that players be in equilibrium so their beliefs are correct with the requirement that their expectations be based on the $k_{1}$ assumption that the opponent will play according to his historical distribution. ${ }^{34}$ We refer to this modified version of quantal response equilibrium as "non-equilibrium quantal response;" it has been used in a variety of economic contexts (see [41] and cites therein).

In this context, players doing one iteration of reasoning play as if the payoff to throwing rock on throw $i$ were

$$
U_{i}^{\text {rock }}=\alpha^{\text {rock }}+\beta \cdot\left(o p p_{i}^{\text {scissors }}-o p p_{i}^{\text {paper }}\right)+\epsilon_{i}^{\text {rock }}
$$

where $o p p^{j}$ is the fraction of the opponents' previous 1st throws that were of type $j$ and $\epsilon_{i} \mathrm{~s}$ are logit errors. (The payoffs for paper and scissors are analogous.) The probability of playing a given throw is increasing in the assumed expected return to that throw. This smooths the threshold response of the

34 If we allowed the expected payoff to vary or be estimated from the data, this would increase the flexibility of the model, making any improvement in fit suspect. However, since the expected payoff is completely determined by the opponent's historical distribution the model has only 3 free parameters-estimated separately for each player-the $\beta$ and two $\alpha$ s. (The third $\alpha$ is normalized to zero.) 
$k_{1}$ strategy into a more continuous response. ${ }^{35}$ The naive version would be to play as if the payoff to throwing rock on throw $i$ were

$$
U_{i}^{\text {rock }}=\alpha^{\text {rock }}+\beta \cdot o p p_{i}^{\text {scissors }}+\epsilon_{i}^{\text {rock }}
$$

We estimate the parameters separately for each player. Figure 7 shows the distribution of the $\beta$ coefficient across individuals. The coefficients for the naive model are higher on average and more dispersed than the baseline. The mean coefficient for the baseline (naive) model is $1.42(2.47){ }^{36}$ The expected return is the probability of winning minus the probability of losing (probability of winning), so it ranges from -1 to 1 ( 0 to 1$)$. The standard deviation is $0.232(0.137)$, so, on average, a standard deviation increase in the expected return to an action, increases the percent chance it is played by approximately 7.3 percentage points ( 7.5 percentage points). ${ }^{37}$ The standard deviation across experienced players in the percent of the time they play a throw is $5 \%$, so this effect is significant, but not huge.

The coefficient on expected return is significant for $63 \%(65 \%)$ of players. The mean of the effect size conditional on being significant is 2.10 (3.56). Converting to margins, this corresponds to a standard deviation increase in expected return resulting in an 11 percentage point increase in the probability of playing a given throw, which is quite large.

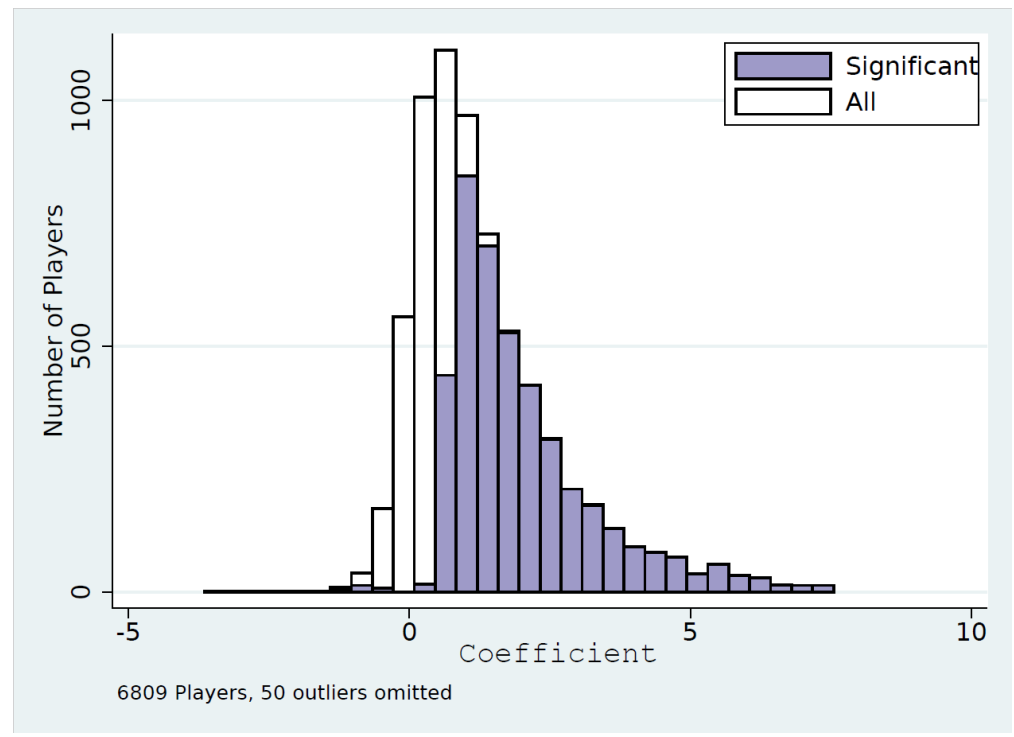

(a) Baseline

Figure 7. Cont.

35 A second level of reasoning would expect opponents to play according to the distribution induced by one's own history and would play with probabilities proportional to the expected payoff against that distribution. However, given the low levels of $k_{2}$ play we find and the econometric difficulties of including own history in the logit, we only analyze the first iteration of reasoning.

36 This suggests that players do respond to expected payoffs calculated from historical opponent play; whereas in the reduced-form results (Table 4 ) we showed that players did not respond to the expected payoff calculated from predicted opponent play-predicted based on the coefficients from Table 3 and players' histories.

37 We multiply by 100 to convert to percentages and by $2 / 9$ to evaluate the margin at the mean: $0.232 \cdot 1.42 \cdot 100 \cdot \frac{2}{9}=7.3$. 


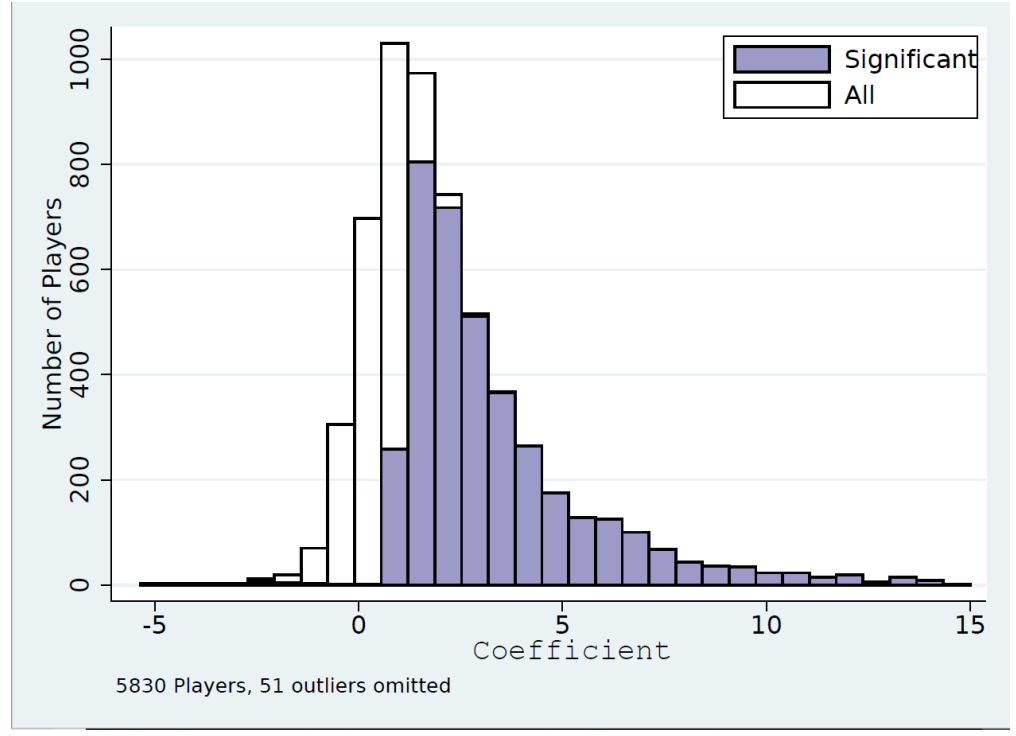

(b) Naive

Figure 7. Distribution across players of the coefficient in the quantal response model. Note: The coefficient is $\beta$ from the logit estimation, run separately for each player, $U_{i}^{\text {rock }}=\alpha^{\text {rock }}+\beta \cdot \mathrm{EP}^{\text {rock }}+\epsilon_{i}^{\text {rock }}$, analogously for paper and scissors, where EP (the expected payoff) for the baseline model is the probability of winning against the opponent's historical distribution minus the probability of losing; for the naive model is just the probability of winning. Outliers more than 4 standard deviations from the mean are omitted.

\section{Likelihood Comparison}

Which is a better model of player behavior, the discrete "if it is the $k_{1}$ throw, play it" or the more continuous "if its $k_{1}$ payoff is higher, play it with higher probability"? Since the strategies are similar, if players were using one there would still be some evidence for the other, so we use a likelihood test to see which model better fits players' behavior. To facilitate the comparison, we estimate a "level- $k_{1}$ " model — the level- $k$ model with no $k_{2}$ - which also has three independent parameters. ${ }^{38}$

As in Section 4.5, if we assume that one of two models, level- $k_{1}(\mathrm{LK})$ and quantal response (QR), generated the data and have a flat prior over which it was, then according to Bayes theorem, the probability that it was quantal response is

$$
P(\mathrm{QR} \mid \text { data })=\frac{P(\text { data } \mid \mathrm{QR})}{P(\text { data } \mid \mathrm{LK})+P(\text { data } \mid \mathrm{QR})} .
$$

We do this calculation for both the standard level- $k$ and the naive level- $k$ (which we compare to an analogous naive $\mathrm{QR}$ model).

Figure 8 plots the distribution of this probability across players. For the baseline strategies, level- $k$ and QR are evenly matched. For $55.7 \%$ of players the QR is a better model; more interestingly, there are substantial numbers of players both to the left of 0.05 and to the right of 0.95 . For 1193 players $(17.88 \%)$ the level $-k_{1}$ model is a statistically better fit and for 1180 players $(17.68 \%)$ the $Q R$ is a statistically better fit. This suggests some players' focus more on whether the throw has the highest expected return $\left(k_{1}\right)$ and other players respond more to the level of the expected return $(\mathrm{QR})$. For the naive strategies, the models do about equally well in the overall population, but for players whose play is significantly

38 Models with additional parameters have more flexibility to fit data better even when the underlying model is no better. We find little evidence of $k_{2}$ and using a $k_{1}$ model avoids choosing how much to penalize the level- $k$ model for the additional parameter. 
more consistent with one of the models, they seem to act on whether a throw is most likely to win rather than to have their play probability be increasing in the probability of winning: for $26.41 \%$ of players the naive level- $k_{1}$ model is a statistically better fit and for $12.25 \%$ of players the naive QR is a statistically better fit.

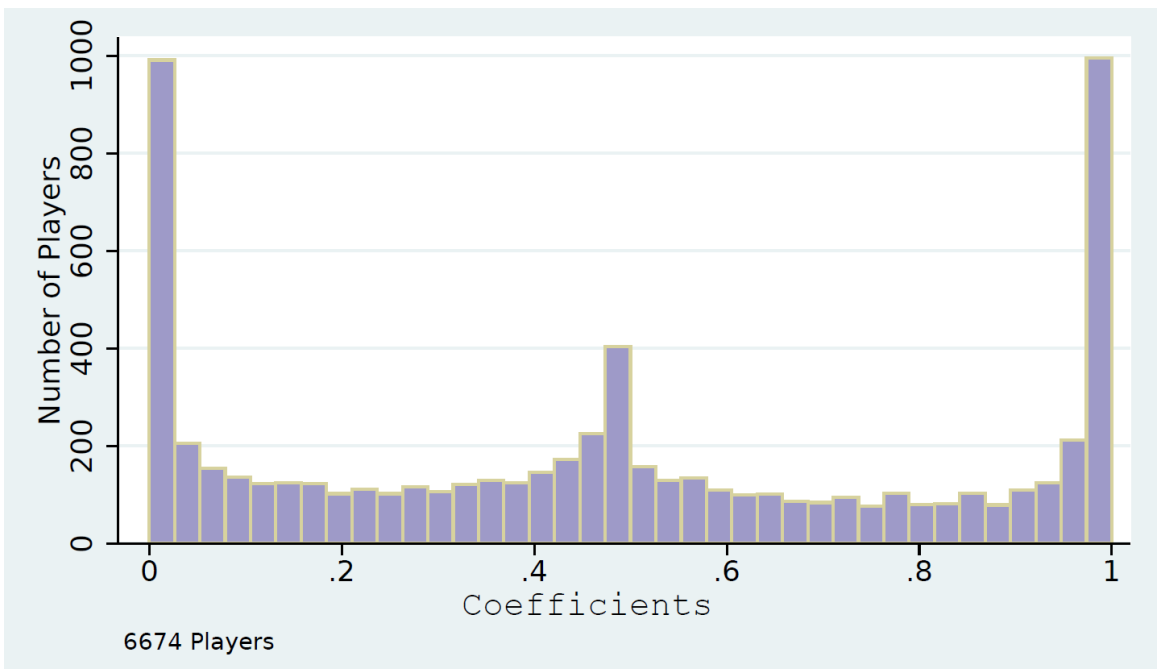

(a) Baseline

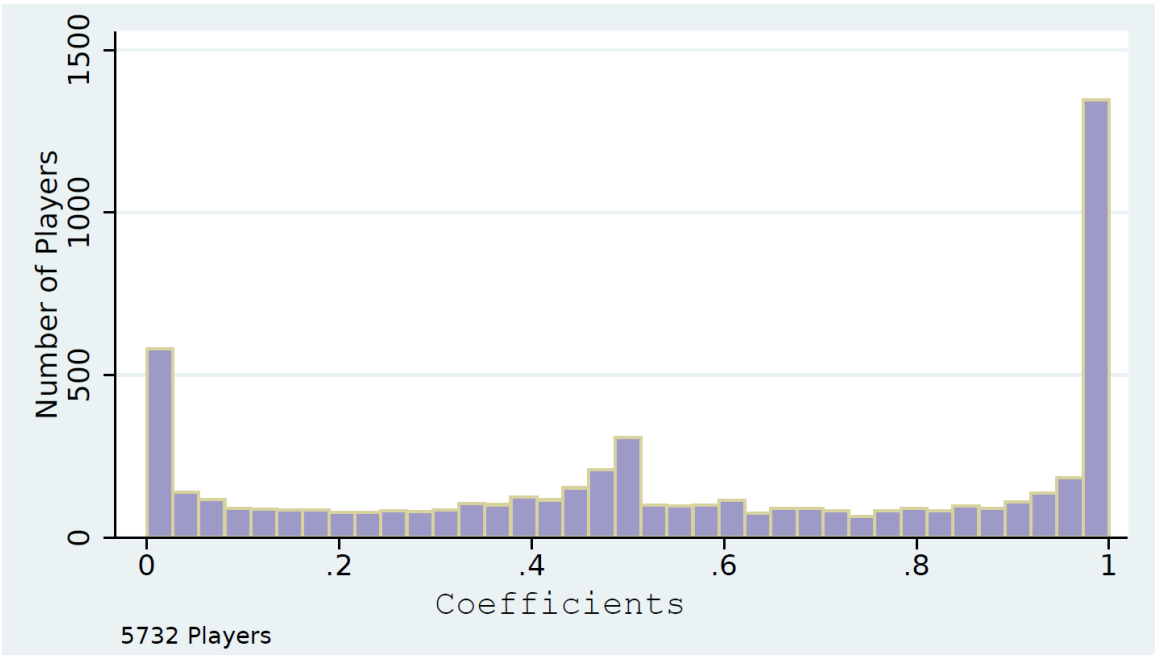

(b) Naive

Figure 8. Probability of the level- $k_{1}$ model. Note: For each player we calculate the probability that the data were generated by the quantal response model as opposed to the level- $k_{1}$ model, assuming a flat prior, as in Equation (2).

We do not have any demographic information on players to look at who tends to use which type of strategy. ${ }^{39}$ However, we can look at whether the number of games they play or their coefficients estimated from the two models predict which model is a better fit. Table 13 presents this analysis, separately for all players and those for whom one model was a significantly better fit. Players who play more games tend to favor the level- $k$ model. Perhaps unsurprisingly, those with a higher estimated fraction of $k_{1}$ play, $\hat{k}_{1}$, and those with a lower $\beta$ coefficient from the QR model also favor the level- $k$ model over the QR.

39 This would be an interesting dimension for future work to explore. 
Table 13. Effect of Players' Parameters on Preferred Model.

\begin{tabular}{|c|c|c|c|c|}
\hline & \multicolumn{2}{|c|}{ All Players } & \multicolumn{2}{|c|}{ Players with Significant Difference } \\
\hline & Regular $k_{1}$ & Naive $k_{1}$ & Regular $k_{1}$ & Naive $k_{1}$ \\
\hline Est. MLE fraction $k_{1}$ play & $\begin{array}{l}3.451^{* * *} \\
(0.0569)\end{array}$ & $\begin{array}{l}2.707^{* * *} \\
(0.0524)\end{array}$ & $\begin{array}{l}3.417^{* * *} \\
(0.0661)\end{array}$ & $\begin{array}{l}2.619^{* * *} \\
(0.0641)\end{array}$ \\
\hline Est. QR coefficient on return to $k_{1}$ & $\begin{array}{c}-0.284^{* * *} \\
(0.0054)\end{array}$ & $\begin{array}{c}-0.106^{* * *} \\
(0.0029)\end{array}$ & $\begin{array}{c}-0.248^{* * *} \\
(0.0051)\end{array}$ & $\begin{array}{c}-0.0870 * * * \\
(0.0027)\end{array}$ \\
\hline Total Number of Matches (100s) & $\begin{array}{c}0.0200 * * * \\
(0.0036)\end{array}$ & $\begin{array}{c}0.0168^{* * * *} \\
(0.0042)\end{array}$ & $\begin{array}{c}0.0238^{* * *} \\
(0.0041)\end{array}$ & $\begin{array}{l}0.0131 \text { * } \\
(0.0051)\end{array}$ \\
\hline Constant & $\begin{array}{l}0.173^{* * *} \\
(0.0106)\end{array}$ & $\begin{array}{l}0.208^{* * *} \\
(0.0120)\end{array}$ & $\begin{array}{c}0.0549 * * \\
(0.0192)\end{array}$ & $\begin{array}{l}0.135^{* * *} \\
(0.0221)\end{array}$ \\
\hline$R^{2}$ & 0.358 & 0.321 & 0.565 & 0.438 \\
\hline $\mathrm{N}$ & 6673 & 5731 & 2373 & 2216 \\
\hline
\end{tabular}

\section{Conclusions}

The 20th century witnessed several breakthrough discoveries in economics. Arguably the most important revolved around understanding behavior in strategic settings, which originated with John von Neumann's (1928) minimax theorem. In zero-sum games with unique mixed-strategy equilibria, minimax logic dictates that strategies should be randomized to prevent exploitation by one's opponent. The work of Nash enhanced our understanding of optimal play in games, and several theorists since have made seminal discoveries.

We continue the empirical work on the topic by analyzing an enormous set of field data on RPS games with information about opponents' past play. In doing so, we can explore the models-both equilibrium and non-equilibrium - that best describe the data. While we find that most people employ strategies consistent with Nash, at least some of the time, there is considerable deviation from equilibrium play. Adapting level- $k$ thinking to our repeated game context, we use maximum likelihood to estimate the frequency with which each player uses $k_{0}, k_{1}$ and $k_{2}$. We find that about three quarters of all throws are best described as $k_{0}$. A little less than one fifth of play is $k_{1}$ or fictitious play, with $k_{2}$ play accounting for less than one-tenth of play. Interestingly, we find that most players are mixing over at least two levels of reasoning. A model where players focus on winning, without worrying about losing, suggests similar levels for each strategy and is a better fit for most players. Since players mix across levels, we explore when they are most likely to play $k_{1}$. We find that consistency with $k_{1}$ is increased when the expected return to $k_{1}$ is higher.

We also explore the $\mathrm{QR}$ model. Our adapted version of $\mathrm{QR}$ has players paying attention to the expected return to each strategy. We find that a one-standard-deviation increase in expected return increases the probability of a throw by 7.3 percentage points. In addition, for about a fifth of players the QR model fits significantly better than the level- $k$ model, but for another one fifth the level- $k$ model fits significantly better. It seems that some players focus on the levels of the expected returns, while others focus on which throw has the highest expected return.

There are several limitations to our analysis, which would be interesting to explore in future work. Examining play beyond the first throw could shed light on convergence of strategies within a match. Our dataset does not include demographic information on the players, so we could not analyze the interaction between strategies and player characteristics. The differences in strategic choices, though, lead to additional questions: who are the players who employ more advanced strategies, and why? Finally, there are other simple games that may lend themselves better to level- $k$ models, including many variants of RPS with more strategies available to the players and with different payoff structures. 
Beyond theory testing, we draw several methodological lessons. First, while our setting is very different from the single-shot games for which that level- $k$ theory was initially developed, our finding that players mix across strategies raises questions for experiments that attempt to categorize players as a $k$-type based on only a few instances of play. Second, with large data sets, subtle differences in theoretical predictions can be tested with meaningful power. As the Internet continues to provide unique opportunities for such large-scale data, we hope that our study can serve as a starting point for future explorations of behavior in both strategic and non-strategic settings.

Author Contributions: Conceptualization, S.L. and J.A.L.; Formal analysis, D.B., S.J. and J.P.; Methodology, D.B. and S.J.; Supervision, S.L. and J.A.L.; Writing—original draft, S.J., S.L. and J.P.; Writing-review \& editing, D.B., S.J., S.L., J.A.L. and J.P.

Funding: No funding was received specifically for this research. Jaffe gratefully acknowledges the support of a National Science Foundation Graduate Research Fellowship.

Conflicts of Interest: The authors declare no conflict of interest.

\section{Appendix A. Behavior in Strategic Settings: Evidence from a Million Rock-Paper-Scissors Games}

\section{Appendix A.1. Proof of Proposition 1}

We formalize the process of playing rock-papers-scissors over Facebook as a sequence of best-of-three matches described by the game $\Gamma$ nested inside a larger game $\hat{\Gamma}$, which includes the matching process that pairs the players. We do not specify the matching process, as it turns out that it does not matter and the following holds for any matching process. ${ }^{40}$ Players may exit the game (and exit may not be random) after any subgame, but not in the middle of one. All players have symmetric payoffs and discount factor $\delta$ across subgames. Our results are similar to Wooders and Shachat [42] for two-outcome games.

Each nested game $\Gamma$ is a "best-of-three" match of RPS played in rounds, which we will call "throws". For each throw, both players simultaneously choose actions $a \in A=\{r, p, s\}$ and the outcome for each player is a win, loss, or tie; $r$ beats $s$, s beats $p$, and $p$ beats $r$. A player wins $\Gamma$ by winning two throws. The winner of $\Gamma$ receives a payoff of 1 and the loser gets -1 . Please note that $\Gamma$ is zero-sum. Therefore, at any stage of $\hat{\Gamma}$ the sum across players of all future discounted payoffs is zero.

Each match consists of at least two throws. Because of the possibility of ties, there is no limit on the length of a match. Let

$$
\mathcal{K}^{l}=\underbrace{A^{2} \times A^{2} \ldots \times A^{2}}_{l \text { times }}
$$

be the set of all possible sequences of $l$ throws by two players. Let $K^{l} \subset \mathcal{K}^{l}$ be the set of possible complete matches of length $l$ : sequences of throw pairs such that no player had 2 wins after $l-1$ throws, but a player had two wins after the $l^{\text {th }}$ throw. Let $K=\cup K^{l}$ be the set of possible complete matches of any length. Let $\hat{K}^{l} \subset \mathcal{K}^{l}$ be the set of possible incomplete matches of length $l$ : sequences of throw pairs such that no player has 2 wins.

A player's overall history after playing $t$ matches is the sequence of match histories for all matches he has played,

$$
h_{i}^{t} \in H^{t}=\underbrace{K \times K \ldots \times K}_{\text {t times }} .
$$

Let $H_{-i}$ denote the history of all players in the pool other than $i$. Players may not observe their opponents' exact histories. Instead a player observes some public summary information of his

40 In the actual game players could challenge a specific player to a game or be matched by the software to someone else who was looking for an opponent. 
opponent's history. Let $f: H^{t} \rightarrow S^{t} \forall t$ be the function that maps histories into summary information. Denote by $s^{t}$ an element of $S^{t}$.

A strategy for a player is a mapping from any history of the game so far, own history of previous games, and summary information about an opponent's history to a distribution of actions. $\sigma$ : $H^{t} \times S^{r} \times \hat{K}^{l} \rightarrow \Delta A$. The set of all players strategies is $\propto$.

It is helpful to define a function \#win $: \hat{K}^{l} \cup K^{l} \rightarrow\{0,1,2\} \forall l$, which denotes the number of wins for player $i$ after a match history. Similarly \#win $n_{j}$ is the number of wins for player $j$ and $\#$ win $=\max \left\{\# w_{i n}, \# w_{i n}\right\}$ is the number of wins of the player with the most wins.

In Nash equilibrium, at any throw of any match, the distribution chosen must maximize the EP. The payoff consists of the flow payoff plus the continuation value from future matches if the match ends on this throw, or the EP from continuing the match with the updated match history if the match does not end on this throw. Let $\eta\left(h_{i}^{t}, \propto\right)$ be the value of being in the pool (and playing future matches) with a history $h_{i}^{t_{i}}$, given all players' strategies and $v_{i}\left(k_{i j}^{l}, h_{i}^{t_{i}}, s_{j}^{t_{j}}, \boldsymbol{\infty}\right)$ be the value (including future matches) of a player $i$ during a match against player $j$ if his history is $h_{i}^{t_{i}}$, his signal about his opponent is $s_{j}^{t_{j}}$, and the match so far is $k_{i j}^{l}$. Both $\eta(\cdot)$ and $v(\cdot)$ could depend on the set of histories of other players the pool, but we do not include that notation because what follows holds for any such set. An equilibrium strategy must satisfy

$$
\sigma_{i}\left(\hat{k}_{i j}^{l}, h_{i}^{t_{i}}, s_{j}^{t_{j}}\right) \in \arg \max _{\sigma_{i}} \mathbb{E}_{\sigma_{i}, \sigma_{j}}\left[u_{i}\left(\left\{\hat{k}_{i j}^{l},\left(a_{i}, a_{j}\right)\right\}, h_{i}^{t_{i}}, s_{j}^{t_{j}}, \boldsymbol{\infty}\right)\right],
$$

where

$$
\begin{aligned}
u\left(\left\{\hat{k}_{i j^{\prime}}^{l}\left(a_{i}, a_{j}\right)\right\}, h_{i}^{t_{i}}, s_{j}^{t_{j}}, \boldsymbol{\infty}\right)= \\
\qquad \begin{array}{ll}
1+\delta \cdot \eta\left(\left\{h_{i}^{t_{i}},\left\{\hat{k}_{i j^{\prime}}^{l},\left(a_{i}, a_{j}\right)\right\}\right\}, \boldsymbol{\infty}\right) & \# w i n_{i}\left(\left\{\hat{k}_{i j^{\prime}}^{l}\left(a_{i}, a_{j}\right)\right\}\right)=2, \\
-1+\delta \cdot \eta\left(\left\{h_{i}^{t_{i}},\left\{\hat{k}_{i j^{\prime}}^{l},\left(a_{i}, a_{j}\right)\right\}\right\}, \boldsymbol{\infty}\right) & \# w i n_{j}\left(\left\{\hat{k}_{i j^{\prime}}^{l}\left(a_{i}, a_{j}\right)\right\}\right)=2, \\
v_{i}\left(\left\{\hat{k}_{i j^{\prime}}^{l},\left(a_{i}, a_{j}\right)\right\}, h_{i}^{t_{i}}, s_{j}^{t_{j}}, \boldsymbol{\infty}\right) & \# w i n\left(\left\{\hat{k}_{i j^{\prime}}^{l}\left(a_{i}, a_{j}\right)\right\}\right)<2 .
\end{array}
\end{aligned}
$$

In the first two cases there is the immediate payoff from the match ending plus the inter-match continuation value, In the last case there is just the intra-match continuation value, which includes the later inter-match continuation value from when the match eventually ends. The inter-match discount factor is $\delta$, we have implicitly set the intra-match discount factor to $1{ }^{41}$

Because each match is symmetric and zero-sum, the inter-match continuation values are unimportant- -they always equal zero.

Lemma A1. Under Nash equilibrium play, $\eta\left(h^{t}\right)=0 \forall h^{t} \in H^{t} \forall t$.

Proof. Suppose that $\eta\left(h_{i}^{t}\right) \neq 0$. If $\eta\left(h_{i}^{t}\right)<0$, then a player with that history could deviate to always playing $\left(\frac{1}{3}, \frac{1}{3}, \frac{1}{3}\right)$. The player would win half their matches and lose half their matches and have continuation value 0 . If $\eta\left(h_{i}^{t}\right)>0$, then since $\hat{\Gamma}$ is zero-sum at every stage, there must exist $h_{j}^{r}$ such that $\eta\left(h_{j}^{r}\right)<0$, which by the same logic cannot happen in equilibrium.

Since inter-match continuation values are all zero, the intra-match continuation value is just the probability of eventually winning the match minus the probability of eventually losing the match. This means that continuation values are zero-sum: $v_{i}\left(\hat{k}_{i j}, h_{i}, s_{j}\right)=-v_{j}\left(\hat{k}_{i j}, h_{i}, s_{j}\right)$.

41 Because what matters for the result is the symmetry across strategies at all stages, having an intra-match discount factor does not change the result, but substantially complicates the proof. 
The symmetry of the match also implies that regardless of history, whenever players are tied for the number of wins in the match, the continuation value going forward is 0 for both players.

Lemma A2. In Nash equilibrium if \#win $\left(\hat{k}_{i j}^{l}\right)=\#$ win $_{j}\left(\hat{k}_{i j}^{l}\right)$, then $v_{i}\left(\hat{k}_{i j}^{l}, h_{i}, s_{j}\right)=v_{j}\left(\hat{k}_{i j}^{l}, h_{j}, s_{i}\right)=0$.

Proof. Assume \#win ${ }_{i}\left(\hat{k}_{i j}^{l}\right)=\# w_{i n}\left(\hat{k}_{i j}^{l}\right)$. Suppose $v_{i}\left(\hat{k}_{i j}, h_{i}, s_{j}\right) \neq 0$. If $v_{i}\left(\hat{k}_{i j}, h_{i}, s_{j}\right)<0$, player $i$ has a profitable deviation to play $\left(\frac{1}{3}, \frac{1}{3}, \frac{1}{3}\right)$ for the remainder of the match and they will win with probability one half, giving them a continuation value of zero. Similarly for $j$. We therefore have $v_{i}\left(\hat{k}_{i j}, h_{i}, s_{j}\right) \geq 0$ and $v_{j}\left(\hat{k}_{i j}^{l}, h_{j}, s_{i}\right) \geq 0$ for all match histories.

If $v_{i}\left(\hat{k}_{i j}^{l}, h_{i}, s_{j}\right)>0$ then since the match is zero-sum, there must exist an $\hat{h}_{i}, \hat{h}_{j}$ such that $v_{i}\left(\hat{k}_{i j}^{l}, \hat{h}_{i}, f\left(\hat{h}_{j}\right)\right)<0$ or $v_{j}\left(\hat{k}_{i j}^{l}, \hat{h}_{j}, f\left(\hat{h}_{i}\right)\right)<0$, which contradicts the above result. Similarly for $v_{j}>0$.

Using this result, we can calculate the continuation when $i$ and $j$ do not have an equal number of wins.

Lemma A3. In Nash equilibrium, if \#win $\hat{k}_{i}\left(\hat{k}_{i j}^{l}\right)=1$ and \#win ${ }_{j}\left(\hat{k}_{i j}^{l}\right)=0$ then $v_{i}\left(\hat{k}_{i j}^{l}, h_{i}, s_{j}\right)=\frac{1}{2}$ and $v_{j}\left(\hat{k}_{i j}^{l}, h_{j}, s_{i}\right)=-\frac{1}{2}$.

Proof. Assume \#win $\left(\hat{k}_{i j}^{l}\right)=1$ and \#win $\left(\hat{k}_{i j}^{l}\right)=0$. Suppose $v_{i}\left(\hat{k}_{i j}^{l}, h_{i}, s_{j}\right)<\frac{1}{2}$. This implies that player $i$ has a profitable deviation to play $\left(\frac{1}{3}, \frac{1}{3}, \frac{1}{3}\right)$ for the remainder of the match. Denote by $x$, the probability that player $i$ wins when play $\left(\frac{1}{3}, \frac{1}{3}, \frac{1}{3}\right)$. They win, lose, or draw on the next throw with equal probability; if they win the next throw, they win the match, if they lose the next throw, they have a $1 / 2$ probability of winning the match and if there is a draw, they are back in the same situation of having won 1 throw and $j$ has won zero, so they will win with probability $x$. So

$$
x=\underbrace{\frac{1}{3}}_{\text {win this throw }} \cdot 1+\underbrace{\frac{1}{3}}_{\text {lose this throw }} \cdot \frac{1}{2}+\underbrace{\frac{1}{3}}_{\text {draw this throw }} \cdot x,
$$

which implies that player $i$ will win with probability $\frac{3}{4}$ and lose with probability $\frac{1}{4}$, giving them an intra-match continuation value of $\frac{1}{2}$. Therefore $v_{i}\left(\hat{k}_{i j}^{l}, h_{i}, s_{j}\right) \geq \frac{1}{2}$. Similar logic guarantees that $v_{j}\left(\hat{k}_{i j}^{l}, h_{j}, s_{i}\right) \geq-\frac{1}{2}$.

If $v_{i}\left(\hat{k}_{i j}^{l}, h_{i}, s_{j}\right)>\frac{1}{2}$ then since the match is zero-sum, there must exist an $\hat{h}_{i}, \hat{h}_{j}$ such that $v_{i}\left(\hat{k}_{i j}^{l}, \hat{h}_{i}, f\left(\hat{h}_{j}\right)\right)<\frac{1}{2}$ or $v_{j}\left(\hat{k}_{i j}^{l}, \hat{h}_{j}, f\left(\hat{h}_{i}\right)\right)<-\frac{1}{2}$, which contradicts the above result. Similarly for $v_{j}\left(\hat{k}_{i j}^{l}, h_{j}, s_{i}\right)>-\frac{1}{2}$.

Since the number of wins each player has after any history that has not ended the match is either 0 or 1, Lemmas A2 and A3 encompass all the possible $\hat{k}_{i, j}^{l}$. We therefore see that the continuation value depends only on the number of wins each player has

$$
v_{i}\left(\hat{k}_{i, j}^{l}, h_{i}, s_{j}\right)=\tilde{v}_{i}\left(\# w i n_{i}\left(\hat{k}_{i, j}^{l}\right), \# \operatorname{win}_{j}\left(\hat{k}_{i, j}^{l}\right)\right) .
$$

Therefore, for every $\hat{k}_{i, j}^{l}$ we can calculate $u_{i}\left(\left\{\hat{k}_{i j}^{l},\left(a_{i}, a_{j}\right)\right\}, h_{i}^{t_{i}}, s_{j}^{t_{j}}\right)$ for each own throw and opponent throw. Figure A1 gives these payoffs for each possible stage in a match. For each of these stages, it can only be a Nash equilibrium if each player expects their opponent to play $\left(\frac{1}{3}, \frac{1}{3}, \frac{1}{3}\right)$. 


\begin{tabular}{c|c|c|c}
$\mathrm{A} \backslash \mathrm{B}$ & $\mathrm{R}$ & $\mathrm{P}$ & $\mathrm{S}$ \\
\hline $\mathrm{R}$ & 0,0 & $-\frac{1}{2}, \frac{1}{2}$ & $\frac{1}{2},-\frac{1}{2}$ \\
\hline $\mathrm{P}$ & $\frac{1}{2},-\frac{1}{2}$ & 0,0 & $-\frac{1}{2}, \frac{1}{2}$ \\
\hline $\mathrm{S}$ & $-\frac{1}{2}, \frac{1}{2}$ & $\frac{1}{2},-\frac{1}{2}$ & 0,0
\end{tabular}

(a) Start of Match

\begin{tabular}{c|c|c|c}
$\mathrm{A} \backslash \mathrm{B}$ & $\mathrm{R}$ & $\mathrm{P}$ & $\mathrm{S}$ \\
\hline $\mathrm{R}$ & $-\frac{1}{2}, \frac{1}{2}$ & $-1,1$ & 0,0 \\
\hline $\mathrm{P}$ & 0,0 & $-\frac{1}{2}, \frac{1}{2}$ & $-1,1$ \\
\hline $\mathrm{S}$ & $-1,1$ & 0,0 & $-\frac{1}{2}, \frac{1}{2}$
\end{tabular}

(c) B has 1 win, A has zero

\begin{tabular}{c|c|c|c}
$\mathrm{A} \backslash \mathrm{B}$ & $\mathrm{R}$ & $\mathrm{P}$ & $\mathrm{S}$ \\
\hline $\mathrm{R}$ & $\frac{1}{2},-\frac{1}{2}$ & 0,0 & $1,-1$ \\
\hline $\mathrm{P}$ & $1,-1$ & $\frac{1}{2},-\frac{1}{2}$ & 0,0 \\
\hline $\mathrm{S}$ & 0,0 & $1,-1$ & $\frac{1}{2},-\frac{1}{2}$
\end{tabular}

(b) A has 1 win, B has zero

\begin{tabular}{c|c|c|c}
$\mathrm{A} \backslash \mathrm{B}$ & $\mathrm{R}$ & $\mathrm{P}$ & $\mathrm{S}$ \\
\hline $\mathrm{R}$ & 0,0 & $-1,1$ & $1,-1$ \\
\hline $\mathrm{P}$ & $1,-1$ & 0,0 & $-1,1$ \\
\hline $\mathrm{S}$ & $-1,1$ & $1,-1$ & 0,0
\end{tabular}

(d) A has 1 win, B has 1 win

Figure A1. Total payoffs (flow + continuation) for each stage of the match.

Appendix A.2. Additional Tables

Table A1. Response to Percent Paper.

\begin{tabular}{ccccc}
\hline \multirow{2}{*}{ Opp's Historical \% } & \multicolumn{3}{c}{ Throws (\%) } & \multirow{2}{*}{$N$} \\
\cline { 2 - 4 } & Paper & Rock & Scissors & \\
\hline $0 \%-25 \%$ & 38.88 & 38.26 & 22.87 & 904,003 \\
$25 \%-30 \%$ & 36.93 & 39.04 & 24.03 & 568,683 \\
$30 \%-33 \frac{1}{3} \%$ & 35.95 & 37.56 & 26.49 & 475,438 \\
$33 \frac{1}{3} \%-37 \%$ & 35.23 & 34.39 & 30.37 & 888,846 \\
$37 \%-42 \%$ & 32.86 & 29.76 & 37.39 & 671,106 \\
$42 \%-100 \%$ & 29.91 & 28.03 & 42.06 & $1,173,439$ \\
\hline
\end{tabular}

Note: Matches are binned by the opponent's historical percentage paper on first throws. For each range of opponent's historical percentage paper, this table shows the distribution of rock, paper, and scissors throws the players use.

Table A2. Response to Percent Scissors.

\begin{tabular}{ccccc}
\hline \multirow{2}{*}{ Opp's Historical \% } & \multicolumn{3}{c}{ Throws (\%) } & \multirow{2}{*}{$N$} \\
\cline { 2 - 4 } & Paper & Rock & Scissors & \\
\hline $0 \%-25 \%$ & 39.61 & 28.05 & 32.34 & $1,308,484$ \\
$25 \%-30 \%$ & 37.96 & 28.07 & 33.98 & 730,391 \\
$30 \%-33 \frac{1}{3} \%$ & 35.92 & 29.67 & 34.41 & 546,698 \\
$33 \frac{1}{3} \%-37 \%$ & 32.85 & 34.29 & 32.86 & 825,972 \\
$37 \%-42 \%$ & 28.77 & 40.99 & 30.24 & 513,185 \\
$42 \%-100 \%$ & 27.24 & 46.64 & 26.12 & 756,785 \\
\hline
\end{tabular}

Note: Matches are binned by the opponent's historical percentage scissors on first throws. For each range of opponent's historical percentage scissors, this table shows the distribution of rock, paper, and scissors throws the players use. 
Table A3. Probability of Playing Rock.

\begin{tabular}{lccc}
\hline Covariate & \multicolumn{2}{c}{ Dependent Var: } & Dummy for Throwing Rock \\
\hline & $(1)$ & $(2)$ & $(3)$ \\
Opp's Fraction Paper (first) & $-0.0403^{* * *}$ & $-0.0718^{* * *}$ & $-0.0952^{* * *}$ \\
& $(0.0021)$ & $(0.0056)$ & $(0.0087)$ \\
Opp's Fraction Scissors (first) & $0.2375^{* * *}$ & $0.1552^{* * *}$ & $0.1377^{* * *}$ \\
& $(0.0022)$ & $(0.0060)$ & $(0.0094)$ \\
Opp's Fraction Paper (all) & 0.0022 & $0.0249^{* *}$ & 0.0032 \\
& $(0.0032)$ & $(0.0088)$ & $(0.0138)$ \\
Opp's Fraction Scissors (all) & $0.0403^{* * *}$ & $0.0238^{*}$ & -0.0187 \\
& $(0.0033)$ & $(0.0093)$ & $(0.0146)$ \\
Opp's Paper Lag & $0.0043^{* * *}$ & -0.0016 & $-0.0043^{*}$ \\
& $(0.0007)$ & $(0.0015)$ & $(0.0019)$ \\
Opp's Scissors Lag & $0.0142^{* * *}$ & $0.0052^{* *}$ & -0.0018 \\
& $(0.0008)$ & $(0.0016)$ & $(0.0020)$ \\
Own Fraction Paper (first) & $-0.0657^{* * *}$ & $0.0290^{* * *}$ & $-0.1092^{* * *}$ \\
& $(0.0020)$ & $(0.0073)$ & $(0.0176)$ \\
Own Fraction Scissors (first) & $-0.0816^{* * *}$ & 0.0013 & $-0.3216^{* * *}$ \\
& $(0.0021)$ & $(0.0076)$ & $(0.0180)$ \\
Own Fraction Paper (all) & $-0.0184^{* * *}$ & $-0.0551^{* * *}$ & $-0.1408^{* * *}$ \\
& $(0.0030)$ & $(0.0113)$ & $(0.0244)$ \\
Own Fraction Scissors (all) & $-0.0097^{* *}$ & $-0.0781^{* * *}$ & $-0.06911^{* *}$ \\
& $(0.0032)$ & $(0.0119)$ & $(0.0253)$ \\
Own Paper Lag & $-0.0015^{*}$ & $0.0121^{* * *}$ & $0.0046^{*}$ \\
& $(0.0007)$ & $(0.0015)$ & $(0.0019)$ \\
Own Scissors Lag & $0.0031^{* * *}$ & $0.0094^{* * *}$ & $-0.0059^{* * *}$ \\
& $(0.0008)$ & $(0.0016)$ & $(0.0019)$ \\
Constant & $0.3423^{* * *}$ & $-0.0190^{* * *}$ & 0.0001 \\
& $(0.0007)$ & $(0.0014)$ & $(0.0018)$ \\
\hline$R^{2}$ & 0.0184 & & \\
$N$ & $4,433,260$ & & \\
\hline
\end{tabular}

Note: $* * *$, and ${ }^{* *}$ indicate significance at the $10 \%, 5 \%$, and $1 \%$ level respectively. This table adds additional variables for a player's own history to the specification in Table 3.

Table A4. Probability of Playing Paper.

\begin{tabular}{lccc}
\hline Covariate & \multicolumn{3}{c}{ Dependent Var: } \\
\hline & $(1)$ & $(2)$ & $(3)$ \\
Opp's Fraction Rock (first) & $0.2554^{* * *}$ & $0.1441^{* * *}$ & $0.1249^{* * *}$ \\
& $(0.0021)$ & $(0.0058)$ & $(0.0091)$ \\
Opp's Fraction Scissors (first) & $-0.0363^{* * *}$ & $-0.0648^{* * *}$ & $-0.1095^{* * *}$ \\
& $(0.0022)$ & $(0.0060)$ & $(0.0095)$ \\
Opp's Fraction Rock (all) & $0.0682^{* * *}$ & 0.0109 & 0.0054 \\
& $(0.0032)$ & $(0.0091)$ & $(0.0144)$ \\
Opp's Fraction Scissors (all) & $-0.0079^{*}$ & -0.0090 & 0.0070 \\
& $(0.0034)$ & $(0.0096)$ & $(0.0153)$ \\
Opp's Rock Lag & $0.0112^{* * *}$ & $0.0048^{* *}$ & $-0.0047^{*}$ \\
& $(0.0007)$ & $(0.0016)$ & $(0.0020)$ \\
Opp's Scissors Lag & $0.0037^{* * *}$ & -0.0029 & $-0.0051^{* *}$ \\
& $(0.0007)$ & $(0.0016)$ & $(0.0019)$ \\
Own Rock Lag & $-0.0070^{* * *}$ & $0.0102^{* * *}$ & $-0.0046^{*}$ \\
& $(0.0007)$ & $(0.0015)$ & $(0.0019)$ \\
Own Scissors Lag & $0.0023^{* * *}$ & $0.0127^{* * *}$ & 0.0012 \\
& $(0.0007)$ & $(0.0015)$ & $(0.0019)$ \\
Constant & $0.3365^{* * *}$ & $-0.0097^{* * *}$ & $0.0065^{* * *}$ \\
& $(0.0006)$ & $(0.0014)$ & $(0.0018)$ \\
\hline$R^{2}$ & 0.0216 & & \\
$N$ & $4,433,260$ & & \\
\hline
\end{tabular}

Note: ***, and ${ }^{* * *}$ indicate significance at the $10 \%, 5 \%$, and $1 \%$ level respectively. All coefficients from one regression. See Table 3 for details. 
Table A5. Probability of Playing Scissors.

\begin{tabular}{lccc}
\hline Covariate & \multicolumn{2}{c}{ Dependent Var: Dummy for Throwing Scissors } \\
\hline & $(1)$ & $(2)$ & $(3)$ \\
Opp's Fraction Paper (first) & $0.2395^{* * * *}$ & $0.1637^{* * *}$ & $0.1241^{* * *}$ \\
& $(0.002)^{* * *}$ & $(0.0061)$ & $(0.0095)$ \\
Opp's Fraction Rock (first) & $-0.0541^{* * *}$ & $-0.0533^{* * *}$ & $-0.0963^{* * *}$ \\
& $\left(0.0021^{* * *}\right.$ & $(0.0057)$ & $(0.0090)$ \\
Opp's Fraction Paper (all) & $0.0326^{* * *}$ & -0.0118 & -0.0171 \\
& $\left(0.0033^{* * *}\right.$ & $(0.0095)$ & $(0.0151)$ \\
Opp's Fraction Rock (all) & $-0.0345^{* * *}$ & 0.0031 & -0.0192 \\
& $\left(0.0032^{* * *}\right.$ & $(0.0090)$ & $(0.0143)$ \\
Opp's Paper Lag & $0.0123^{* * *}$ & $0.0045^{* *}$ & -0.0025 \\
& $(0.0007)$ & $(0.0016)$ & $(0.0020)$ \\
Opp's Rock Lag & $0.0063^{* * *}$ & -0.0022 & -0.0021 \\
& $(0.0007)$ & $(0.0016)$ & $(0.0019)$ \\
Own Paper Lag & $0.0049^{* * *}$ & $0.0096^{* * *}$ & $-0.0085 * * *$ \\
& $(0.0007)$ & $(0.0015)$ & $(0.0019)$ \\
Own Rock Lag & $-0.0052^{* * *}$ & $0.0233^{* * *}$ & 0.0011 \\
& $(0.0007)$ & $(0.0015)$ & $(0.0019)$ \\
Constant & $0.3034^{* * *}$ & 0.0000 & $0.0047^{* *}$ \\
& $(0.0006)$ & $(0.0014)$ & $(0.0018)$ \\
\hline$R^{2}$ & 0.0214 & & \\
$N$ & $4,433,260$ & & \\
\hline
\end{tabular}

Note: ${ }^{* *}$, and ${ }^{* * *}$ indicate significance at the $5 \%$ and $1 \%$ level respectively. All coefficients are from one regression. See Table 3 for details.

Table A6. Opponents' Response to Expected Payoff of Paper.

\begin{tabular}{lcccr}
\hline \multirow{2}{*}{ Opponent's Expected Payoff of Paper } & \multicolumn{3}{c}{ Opponent's Throw (\%) } & \multirow{2}{*}{$\boldsymbol{N}$} \\
\cline { 2 - 4 } & Paper & Rock & Scissors & \\
\hline$[-1,-0.666]$ & 33.74 & 34.52 & 31.74 & 2448 \\
{$[-0.666,-0.333]$} & 36.5 & 34.06 & 29.44 & 11,126 \\
{$[-0.333,0]$} & 34.77 & 33.78 & 31.45 & 836,766 \\
{$[0,0.333]$} & 34.27 & 33.38 & 32.35 & $1,358,196$ \\
{$[0.333,0.666]$} & 32.57 & 34.57 & 32.86 & 9867 \\
\hline
\end{tabular}

Note: See the note on Table 4 for how expected payoff is calculated.

Table A7. Opponents' Response to Expected Payoff of Scissors.

\begin{tabular}{lcccr}
\hline \multirow{2}{*}{ Opponent's Expected Payoff of Scissors } & \multicolumn{3}{c}{ Opponent's Throw (\%) } & \multirow{2}{*}{$N$} \\
\cline { 2 - 4 } & Paper & Rock & Scissors & \\
\hline$[-1,-0.666]$ & 34.24 & 32.49 & 33.27 & 1659 \\
{$[-0.666,-0.333]$} & 33.72 & 34.97 & 31.31 & 19,249 \\
{$[-0.333,0]$} & 35.46 & 32.02 & 32.52 & $1,041,591$ \\
{$[0,0.333]$} & 33.6 & 34.81 & 31.59 & $1,139,164$ \\
{$[0.333,0.666]$} & 31.73 & 40.02 & 28.24 & 16,740 \\
\hline
\end{tabular}

Note: See the note on Table 4 for how expected payoff is calculated. 
Table A8. Effect of Expected $k_{2}$ Payoff on $k_{2}$-Consistency (OLS).

\begin{tabular}{|c|c|c|c|}
\hline & (1) & (2) & (3) \\
\hline Payoff from Playing K2 & $\begin{array}{c}-0.0211^{* * *} \\
(0.0010)\end{array}$ & $\begin{array}{l}0.159 * * * \\
(0.0015)\end{array}$ & $\begin{array}{l}0.145^{* * *} \\
(0.0019)\end{array}$ \\
\hline High Opponent Experience & & & $\begin{array}{l}0.005^{* * *} \\
(0.0014)\end{array}$ \\
\hline Medium Opponent Experience & & & $\begin{array}{c}-0.001 \\
(0.0012)\end{array}$ \\
\hline K2 Payoff X High Opponent Experience & & & $\begin{array}{l}0.052^{* * *} \\
(0.0037)\end{array}$ \\
\hline K2 Payoff X Medium Opponent Experience & & & $\begin{array}{l}0.045^{* * *} \\
(0.0029)\end{array}$ \\
\hline Experienced & $\begin{array}{l}0.006^{* * *} \\
(0.0011)\end{array}$ & & \\
\hline Exp X Payoff from Playing K2 & $\begin{array}{c}-0.012 * * \\
(0.0047)\end{array}$ & $\begin{array}{c}-0.085^{* * *} \\
(0.0047)\end{array}$ & $\begin{array}{c}-0.093^{* * *} \\
(0.0065)\end{array}$ \\
\hline Exp X High Opponent Experience & & & $\begin{array}{l}0.006^{* *} \\
(0.0027)\end{array}$ \\
\hline Exp X Medium Opponent Experience & & & $\begin{array}{l}0.008^{* * *} \\
(0.0027)\end{array}$ \\
\hline Exp X K2 Payoff X High Opponent Experience & & & $\begin{array}{c}-0.027 \text { ** } \\
(0.011)\end{array}$ \\
\hline Exp X K2 Payoff X Medium Opponent Experience & & & $\begin{array}{c}-0.036^{* * *} \\
(0.010)\end{array}$ \\
\hline Own Games > 100 & $\begin{array}{c}0.002 \\
(0.0018)\end{array}$ & & \\
\hline Own Games $>100$ X Payoff from Playing K2 & $\begin{array}{c}-0.097^{* * *} \\
(0.021)\end{array}$ & $\begin{array}{c}0.144^{* * *} \\
(0.013)\end{array}$ & $\begin{array}{c}0.119^{* * *} \\
(0.022)\end{array}$ \\
\hline Own Games $>100$ X High Opponent Experience & & & $\begin{array}{c}-0.024^{* * *} \\
(0.0025)\end{array}$ \\
\hline Own Games >100 X Medium Opponent Experience & & & $\begin{array}{c}-0.025^{* * *} \\
(0.0031)\end{array}$ \\
\hline Own Games $>100$ X K2 Payoff X High Opponent Experience & & & $\begin{array}{c}0.170 * * * \\
(0.028)\end{array}$ \\
\hline Own Games >100 X K2 Payoff X Medium Opponent Experience & & & $\begin{array}{c}0.180^{* * *} \\
(0.033)\end{array}$ \\
\hline Player Fixed Effects & No & Yes & Yes \\
\hline Observations & $4,130,024$ & $4,130,024$ & $4,130,024$ \\
\hline Adjusted $R^{2}$ & 0.000 & 0.003 & 0.004 \\
\hline
\end{tabular}

\section{References}

1. Camerer, C. Behavioral Game Theory: Experiments in Strategic Interaction; Princeton University Press: Princeton, NJ, USA, 2003.

2. Kagel, J.H.; Roth, A.E. The Handbook of Experimental Economics; Princeton University Press: Princeton, NJ, USA, 1995.

3. Chiappori, P.A.; Levitt, S.; Groseclose, T. Testing mixed-strategy equilibria when players are heterogeneous: The case of penalty kicks in soccer. Am. Econ. Rev. 2002, 92, 1138-1151. [CrossRef]

4. Frey, B.S.; Meier, S. Social comparisons and pro-social behavior: Testing "conditional cooperation" in a field experiment. Am. Econ. Rev. 2004, 94, 1717-1722. [CrossRef]

5. Karlan, D.S. Using experimental economics to measure social capital and predict financial decisions. Am. Econ. Rev. 2005, 95, 1688-1699. [CrossRef]

6. Walker, M.; Wooders, J. Minimax play at Wimbledon. Am. Econ. Rev. 2001, 91, 1521-1538. [CrossRef] 
7. Hoffman, M.; Suetens, S.; Gneezy, U.; Nowak, M.A. An experimental investigation of evolutionary dynamics in the Rock-Paper-Scissors game. Sci. Rep. 2015, 5, 8817. [CrossRef] [PubMed]

8. Cason, T.N.; Friedman, D.; Hopkins, E. Cycles and instability in a rock-paper-scissors population game: A continuous time experiment. Rev. Econ. Stud. 2013, 81, 112-136. [CrossRef]

9. Brown, G.W. Iterative Solutions of Games by Fictitious Play. In Activity Analysis of Production and Allocation; Koopmans, T., Ed.; Wiley: New York, NY, USA, 1951.

10. Berger, U. Fictitious Play in 2xN Games. J. Econ. Theory 2005, 120, 139-154. [CrossRef]

11. Berger, U. Brown's original fictitious play. J. Econ. Theory 2007, 135, 572-578. [CrossRef]

12. Young, H.P. The Evolution of Conventions. Econometrica 1993, 61, 57-84. [CrossRef]

13. Mookherjee, D.; Sopher, B. Learning Behavior in an Experimental Matching Pennies Game. Games Econ. Behav. 1994, 7, 62-91. [CrossRef]

14. Mookherjee, D.; Sopher, B. Learning and Decision Costs in Experimental Constant Sum Games. Games Econ. Behav. 1997, 19, 97-132. [CrossRef]

15. Stahl, D.O. Evolution of Smart ${ }_{n}$ Players. Games Econ. Behav. 1993, 5, 604-617. [CrossRef]

16. Stahl, D.O.; Wilson, P.W. Experimental evidence on players' models of other players. J. Econ. Behav. Organ. 1994, 25, 309-327. [CrossRef]

17. Stahl, D.O.; Wilson, P.W. On Players' Models of Other Players: Theory and Experimental Evidence. Games Econ. Behav. 1995, 10, 218-254. [CrossRef]

18. Nagel, R. Unraveling in guessing games: An experimental study. Am. Econ. Rev. 1995, 85, 1313-1326.

19. Costa-Gomes, M.; Crawford, V.P. Cognition and behavior in two-person guessing games: An experimental study. Am. Econ. Rev. 2006, 96, 1737-1768. [CrossRef]

20. Crawford, V.P.; Iriberri, N. Fatal attraction: Salience, naivete, and sophistication in experimental "Hide-and-Seek" games. Am. Econ. Rev. 2007, 97, 1731-1750. [CrossRef]

21. Arad, A.; Rubinstein, A. The 11-20 Money Request Game: A Level-k Reasoning Study. Am. Econ. Rev. 2012, 102, 3561-3573. [CrossRef]

22. Costa-Gomes, M.; Crawford, V.P.; Broseta, B. Cognition and Behavior in Normal-Form Games: An Experimental Study. Econometrica 2001, 69, 1193-1235. [CrossRef]

23. Hedden, T.; Zhang, J. What do you think I think you think?: Strategic reasoning in matrix games. Cognition 2002, 85, 1-36. [CrossRef]

24. Ho, T.H.; Camerer, C.; Weigelt, K. Iterated dominance and iterated best response in experimental "p-beauty contests". Am. Econ. Rev. 1998, 88, 947-969.

25. Bosch-Domenech, A.; Montalvo, J.G.; Nagel, R.; Satorra, A. One, two, (three), infinity,...: Newspaper and lab beauty-contest experiments. Am. Econ. Rev. 2002, 92,1687-1701. [CrossRef]

26. Ostling, R.; Wang, J.; Chou, E.; Camerer, C. Testing game theory in the field: Swedish LUPI lottery games. Am. Econ. J. Microecon. 2011, 3, 1-33. [CrossRef]

27. Gillen, B. Identification and estimation of level-k auctions. SSRN Electron. J. 2009. [CrossRef]

28. Goldfarb, A.; Xiao, M. Who thinks about the competition? Managerial ability and strategic entry in US local telephone markets. Am. Econ. Rev. 2011, 101, 3130-3161. [CrossRef]

29. Brown, A.; Camerer, C.; Lovallo, D. To review or not to review? Limited strategic thinking at the movie box office. Am. Econ. J. Microecon. 2012, 4, 1-26. [CrossRef]

30. McKelvey, R.D.; Palfrey, T.R. Quantal response equilibria for normal form games. Games Econ. Behav. 1995, 10, 6-38. [CrossRef]

31. Vogel, C. Rock, Paper, Payoff: Child's Play Wins Auction House an Art Sale. New York Times, 2005. Available online: https:/ / www.nytimes.com/2005/04/29/arts/design/rock-paper-payoff-childs-play-wins-auctionhouse-an-art-sale.html (accessed on 12 December 2013).

32. Facebook. Developer Partner Case Study: Red Bull Application. 2010. Available online: https://allfacebook. de/wp-content/uploads/2010/04/81162-RedBull-Case-Study-FINAL.pdf (accessed on 12 December 2013).

33. Weber, R.A. 'Learning' with no feedback in a competitive guessing game. Games Econ. Behav. 2003, 44, 134-144. [CrossRef]

34. Monderer, D.; Shapley, L.S. Fictitious play property for games with identical interests. J. Econ. Theory 1996, 68, 258-265. [CrossRef]

35. Georganas, S.; Healy, P.J.; Weber, R.A. On the Persistence of Strategic Sophistication. Ohio State Working Paper Series No. 4653. Available online: https:/ / ssrn.com/abstract=2407895 (accessed on 15 May 2014). 
36. Kline, B. An Empirical Model of Non-Equilibrium Behavior in Games. Quant. Econ. 2018, 9, $141-181$. [CrossRef]

37. Hahn, P.R.; Goswami, I.; Mela, C. A Bayesian hierarchical model for inferring player strategy types in a number guessing game. arXiv 2014, arXiv:1409.4815.

38. Houser, D.; Keane, M.; McCabe, K. Behavior in a dynamic decision problem: An analysis of experimental evidence using a Bayesian type classification algorithm. Econometrica 2004, 72, 781-822. [CrossRef]

39. Camerer, C.; Ho, T.H.; Chong, J.K. A cognitive hierarchy model of games. Q. J. Econ. 2004, 119, 861-898. [CrossRef]

40. Wright, J.R.; Leyton-Brown, K. Predicting human behavior in unrepeated, simultaneous-move games. Games Econ. Behav. 2017, 106, 16-37. [CrossRef]

41. Daniel McFadden uantal Choice Analysis: A survey. Ann. Econ. Soc. Meas. 1996, 5, 390-393.

42. Wooders, J.; Shachat, J.M. On the irrelevance of risk attitudes in repeated two-outcome games. Games Econ. Behav. 2001, 34, 342-363. [CrossRef]

(c) 2019 by the authors. Licensee MDPI, Basel, Switzerland. This article is an open access article distributed under the terms and conditions of the Creative Commons Attribution (CC BY) license (http:/ / creativecommons.org/licenses/by/4.0/). 